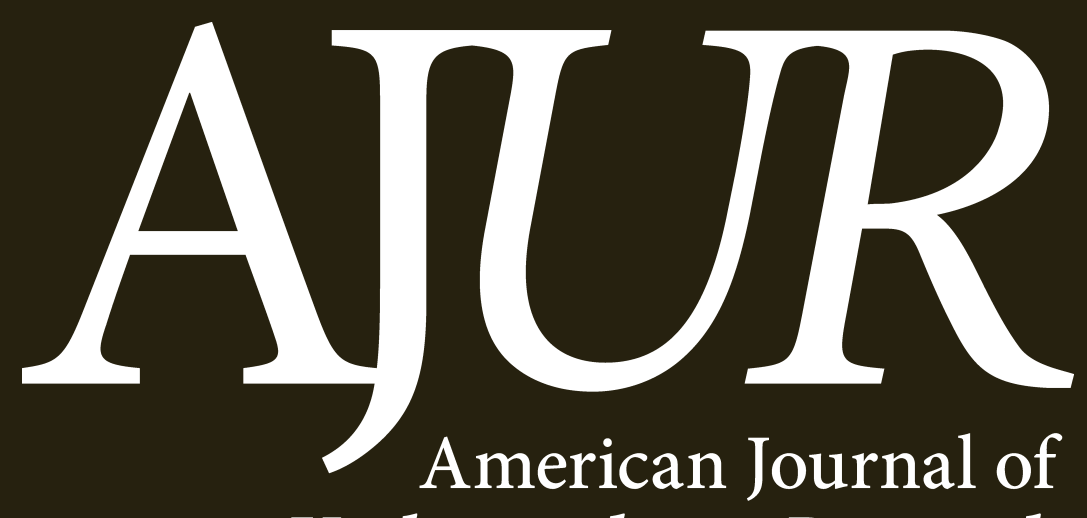
Undergraduate Research

Volume 12 | Issue 1 | August 2014 $w w w . a j u r o n l i n e . o r g$ 



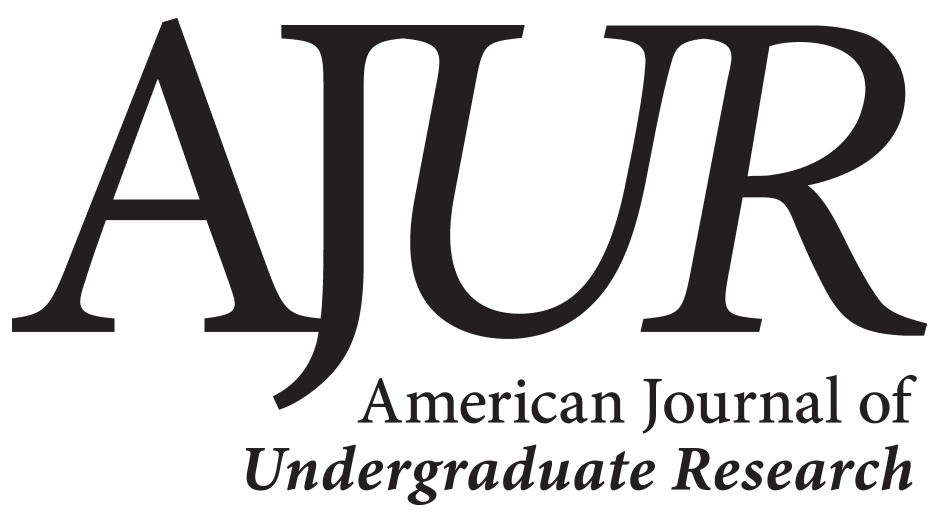

Volume 12 | Issue 1 | August 2014

www.ajuronline.org

2

IN MEMORIAM of Dr. C. Clifton Chancey

3

Special Thanks to AJUR's Sponsors

4

AJUR History and Editorial Board

5 Design Techniques for the DNA Cubic-Lattice

Tyler Hotte \& Miranda LaRocque

20 A Student Approach to a Mathematical Simulation of a Racing Electric Vehicle

Fernando Daniel León-Cázares \& Daniel Xoconostle-Luna

27 Proteomic Study of Ribosomal Proteins from Escherichia coli, Saccharomyces cerevisiae, Bos taurus, Gallus gallus, and Oncorhynchus tshawytscha: Application in a Teaching Laboratory Setting Yoshihiro Miura, Eric Yeager, James A. MacKenzie \& Kestutis Bendinskas

39 A Cellular Automaton Model for Traffic Flow - Investigating the Effect of Turning Tracy Finner \& Matthew A. Beauregard

49 On Deflection of Potentially Dangerous Asteroids Josh Fixelle \& Mikhail Kagan 
The August 2014 issue of AJUR is dedicated to the memory of C. Clifton Chancey.

\section{N M EMOR I A M \\ C. Clifton Chancey (1955-2013)}

Dr. C. Clifton "Cliff" Chancey was born in Cincinnati, Ohio in 1955. He received a B.S. degree in physics from Miami University (Ohio), and M.S. and Ph.D. degrees in physics from The Johns Hopkins University. He spent three years at Oxford University in England as a postdoctoral fellow. He subsequently held appointments at Amherst College, Purdue University-Calumet and the University of Northern Iowa (UNI), where he was professor and head of the Department of Physics until his untimely death in 2013.

Dr. Chancey was a theoretical condensed-matter physicist whose research interests were wideranging. He made contributions in the areas of superconductivity, modeling of sodium channels, sedimentology, and especially the Jahn-Teller Effect. Dr. Chancey was the author or co-author of 27 journal publications. He co-authored (with M. O'Brien) a monograph entitled "The Jahn-Teller Effect in C60 and Other Icosahedral Complexes," published by Princeton University Press in 1997. Dr. Chancey was also the Principal Investigator of research and teaching grants totaling nearly $\$ 3$ million.

Dr. Chancey was an ardent supporter of involving undergraduates in research to develop the skills necessary for the successful practice of science. He involved undergraduates in his own theoretical research. At Purdue-Calumet, he served as the editor of the Northwest Indiana Journal of Undergraduate Research and at UNI, he was the Editor in Chief of the American Journal of Undergraduate Research (this journal). Dr. Chancey's perspective was that the importance of peerreviewed research articles authored by undergraduates could not be overstated.

Dr. Chancey's record of service to the science profession was extensive. He served as Coordinator for the National Task Force for Project Kaleidoscope; he held several national leadership positions in Sigma Xi (The Scientific Research Society), including Director for Comprehensive Colleges and Universities; he was intimately involved in the development and marketing of the Professional Science Master (PSM) degree program in the United States, and served on the Board of Directors of the National PSM Association. Dr. Chancey also served on numerous university committees and was a leader in United Way fundraising campaigns in Cedar Falls, Iowa.

The family, friends and colleagues of Dr. Chancey mourn his passing. He will be sorely missed. 


\section{SPECI A L THANKS}

AJUR is made possible through the assistance of our sponsors.

Support for this issue has been provided by the Office of the Provost, at the State University of New York at Oswego.

Thank you!

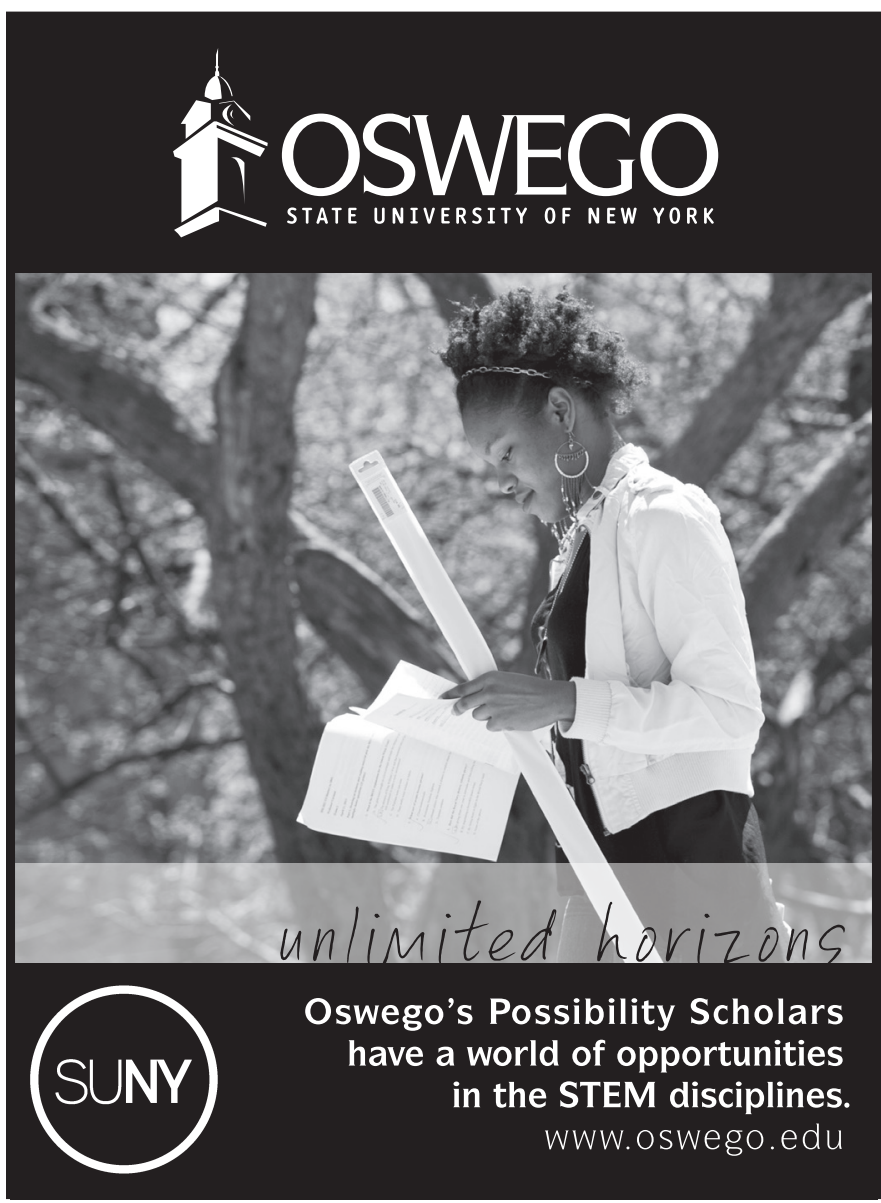

Interested in supporting quality undergraduate research? Request sponsorship information at editor@ajuronline.org 
American Journal of Undergraduate Research (AJUR) is a national, independent, peer reviewed, open-source, just-in-time, multidisciplinary student research journal for excellent student articles.

AJUR (ISSN 1536-4585) was established in 2002 by Dr. Cliff Chancey of the University of Northern Iowa, a true champion of undergraduate research. "Dr. Chancey's perspective was that the importance of peer-reviewed research articles authored by undergraduates could not be overstated"- as noted in this issue's memoriam dedicated to Cliff. In 2013, Dr. Carl N. Drummond and Ms. Cathleen M. Carosella of Indiana University-Purdue University stewarded AJUR.

EDITORIAL TEAM Volume 12 | Issue 1 | August 2014

Dr. Kestutis Bendinskas, Editor, editor@ajuronline.org

Dr. Anthony Contento, Assistant Technical Editor, technicaleditor@ajuronline.org

Rose Throop, Art and Copy Editor,copyeditor@ajuronline.org

Daniel Laird, Web Master,webmaster@ajuronline.org

EDITORIAL BOARD by subject area

ART HISTORY

Dr. Lisa Seppi,

lisa.seppi@oswego.edu

BEHAVIORAL NEUROSCIENCE

Dr. Aileen M. Bailey,

ambailey@smcm.edu

BIOCHEMISTRY

Dr. John A. Bumpus,

john.bumpus@uni.edu

BIOINFORMATICS

Dr. Kevin Daimi,

daimikj@udmercy.edu

Dr. John R. Jungck,

jungck@udel.edu

BIOLOGY, PHYSIOLOGY

Dr. David Dunn,

david.dunn@oswego.ed

BIOLOGY, DEVELOPMENTAL

Dr. Poongodi Geetha-Loganathan,

p.geethaloganathan@oswego.edu

BIOLOGY, MICROBIOLOGY

Dr. Peter Newell,

peter.newell@oswego.edu

BOTANY

Dr. William R. Bromer,

wbromer@stfrancis.edu

CHEMISTRY

Dr Alfredo Castro,

castroa@felician.edu

Dr. Charles Kriley,

cekriley@gcc.edu

Dr. Douglas Mulford,

douglas.mulford@emory.edu

Dr. Mark A. Benvenuto,

benvenma@udmercy.edu
CHEMISTRY, PHYSICAL

Dr. John Oakes,

john.oakes@gcccd.net

COMPUTATIONAL SCIENCES

Dr. Dele Oluwade, deleoluwade@yahoo.com

Dr. J. Ben Schafer,

schafer@cs.uni.edu

ECOLOGY

Dr. William R. Bromer,

wbromer@stfrancis.edu

EDUCATION, PHYSICS

Dr. Andrew D. Gavrin,

agavrin@iupui.edu

ENGINEERING, ENVIRONMENTAL

Dr. Eileen M. Cashman,

eileen.cashman@humboldt.edu

\section{ENVIRONMENTAL SCIENCE}

Dr. Eileen M. Cashman,

eileen.cashman@humboldt.edu

GEOLOGY

Dr. Larry Davis,

ldavis@csbsju.edu

HONORARY EDITORIAL

BOARD MEMBER

Dr. Lorrie Clemo,

lorrie.clemo@oswego.edu

KINESIOLOGY / EXERCISE SCIENCE

Dr. David Senchina,

david.senchina@drake.edu

\section{MATHEMATICS}

Dr. Nkechi M. Agwu,

nagwu@bmcc.cuny.edu

Dr. John Emert,

emert@bsu.edu

Dr. Jeffrey J. Boats,

boatsjj@udmercy.edu

Dr. J.D. Phillips,

jophilli@nmu.edu

Dr. Dele Oluwade,

deleoluwade@yahoo.com

METEOROLOGY

Dr. Steven Skubis,

steven.skubis@oswego.edu

NEUROSCIENCE

Dr. Pamela E. Scott-Johnson, pamela.scottjohnson@morgan.edu

Dr. Amy Overman,

aoverman@elon.edu

\section{PSYCHOLOGY}

Dr. Amy Overman, aoverman@elon.edu

Dr. Pamela E. Scott-Johnson, pamela.scottjohnson@morgan.edu

\section{STATISTICS}

Dr. Mark Ecker, mark.ecker@uni.edu

\section{TECHNOLOGY, ENGINEERING}

Dr. Recayi Pecen,

regpecen@na.edu 


\title{
Design Techniques for the DNA Cubic-Lattice
}

\author{
Tyler Hotte and Miranda LaRocque* \\ Saint Michael's College, Colchester, VT \\ *Students: thotte91@gmail.com, mlarocque@nationallifegroup.com \\ Mentors: jellis0monaghan@smcvt.edu,gpangborn@smcvt.edu
}

\begin{abstract}
We use the Watson-Crick properties of DNA and the principles of graph theory to construct origami folding designs for self-assembling cubic lattices. Our objective is a mathematical design strategy that can be expanded systematically to any size cubic lattice. This design consists of threading a scaffolding strand of DNA through the lattice that is secured in place by short staple strands of DNA. We first add augmenting edges to the cubic lattice to enable a single scaffolding strand threading. We then thread the scaffolding strand through the augmented cube in a way that minimizes the number of different vertex configurations in the structure.
\end{abstract}

\section{KEYWORDS}

Watson-Crick, DNA Self-Assembly, Origami folding, Cubic Lattice, Scaffolding Strand, Threading, Staple Strands

\section{INTRODUCTION}

Recent research has focused on the self-assembly of DNA structures, which can be used for biomolecular computing, targeted drug delivery and biosensors. ${ }^{1}$ There are several structures that have already been created from self-assembling DNA molecules, such as cubes, ${ }^{2}$ truncated octahedra, ${ }^{3}$ rigid octahedra, ${ }^{4}$ tetrahedra, and dodecahedra. ${ }^{5}$ The construction methods of these nanostructures have evolved over time. The branch junction molecule method, or tile based method, previously dominated the field, but as time has progressed, so have the methods of DNA construction. Origami methods have started to become the foremost techniques in the self-assembly of DNA. ${ }^{1}$ This method begins with a single scaffolding strand of DNA that traces the edges of the structure exactly once, and shorter strands bond to this strand to fold and staple it into the desired configuration. The origami method has been specifically used in the construction of nanorobots and carriers, drug and material delivery and directed positioning of particles and materials. ${ }^{1}$

In this paper, we will be working with a design for the cubic lattice. We begin with a description of the design and threading of a 1x1x1 cube. From this we gain insight into cubic lattices of all sizes noting that the first step must be to add augmenting edges so that all vertices have an even degree. We provide three different augmenting strategies, two for $n \times n \times n$ cubes with $n$ odd and one for $n$ even. We then give a complete threading and stapling of the augmented $2 \times 2 \times 2$ and $3 \times 3 \times 3$ cubes. 


\section{DNA BACKGROUND}

Deoxyribonucleic acid (DNA) is typically in a double helix form that is constructed from two separate strands of DNA that are complements to one another. Each strand consists of a backbone made up of sugar and phosphate molecules and nitrogenous bases that hold the information stored in the DNA. The four bases are: Adenine (A), thymine (T), guanine $(\mathrm{G})$ and cytosine $(\mathrm{C})$. These bases pair off with one another to form base pairs that form the treads of the DNA double helix; A must pair with T, and C must pair with G. In order for a strand of DNA to bond with another, the sequences of bases must be perfectly complementary to one another. For example, a strand GTACCTA can only bond with its opposite strand, CATGGAT. When placed in the right solution, the self-assembly properties in DNA will allow a strand to bond together with its complementary bases and form a double strand. ${ }^{6}$

To create the cubic lattice using the method of origami folding, we want to take a single strand of DNA and wrap it around every edge of the structure exactly once. The precise sequence of bases in this strand is either custom designed or comes from an existing virus strand, so we do not specify it here. This strand of DNA is called a scaffolding strand, and the process of wrapping the scaffolding strand around the structure is called a threading. DNA is directional, and therefore its direction must also be accounted for in the design of any structure. For this threading to be possible, we need an Eulerian cycle to be present in the lattice. An Euler cycle is a path that runs through a graph and covers each edge exactly once. Vertices may be visited multiple times, but no edges can be repeated, and the cycle must start and end at the same place. ${ }^{7}$ This is only possible if each vertex in the graph is of even degree. ${ }^{7}$ The degree of a vertex is determined by the number of edges that go into and out of the vertex. Once all vertices have even degree, the scaffolding strand of DNA can be threaded throughout the structure.

In order for the structure to remain in the shape of the lattice, we must attach staple strands into the structure to hold the lattice in its shape. These staples are added at the vertices of the structure to ensure the structure remains intact. The staple strands must be perfect complements to the portions of the scaffolding strands that they hold, and they must be directed in the opposite directions of the threading. The following constraints must be satisfied by the threading and stapling processes.

\section{Threading and stapling constraints:}

- Every edge must be included in the threading exactly once.

- Each vertex must have the same in-degree as out-degree. In other words, we must have the same number of edges entering a vertex as leaving it.

- We can only thread/staple angles of $45^{\circ}$ and $90^{\circ}$, not $180^{\circ}$.

- We cannot cross over any edges while stapling/threading: i.e. a staple or thread should not be forced to leave the plane formed by the two edges it follows by an edge between them.

- Staple strands must be oriented oppositely from the scaffolding strand. 

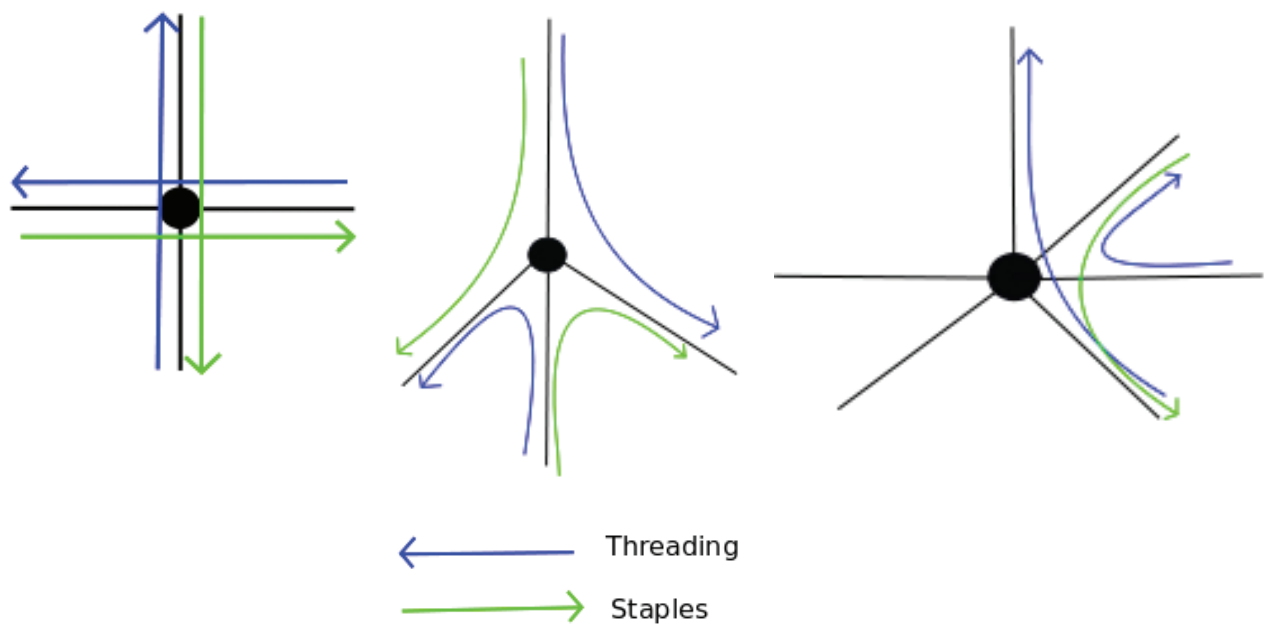

Figure 1. Depiction of prohibited thread and staple configurations. The figure on the left shows a $180^{\circ}$ thread and staple configuration, so the structure would "detach" at this vertex. The center figure shows staples that are traveling in the same direction as the threads. The figure on the right shows 5 coplanar edges, and a sixth vertical edge perpendicular to them, with a staple that has crossed over an edge in the same plane as the stapled edges.

\section{LATTICE BACKGROUND}

A cubic lattice is a structure that is made up of unit-sized cubes. When we talk about cube sizes, we will refer to a cube as size $n$. For example, when we discuss a cube that is $3 \times 3 \times 3$, it will be referred to as a size 3 cube. In order to find an Euler cycle in the lattice, we need each vertex to have even degree. In the case of the cubic lattice, the structure must be modified to meet this constraint. Each corner of the lattice has degree three and all of the vertices in the middle of each face not touching any corner have degree five. (Figure 2)

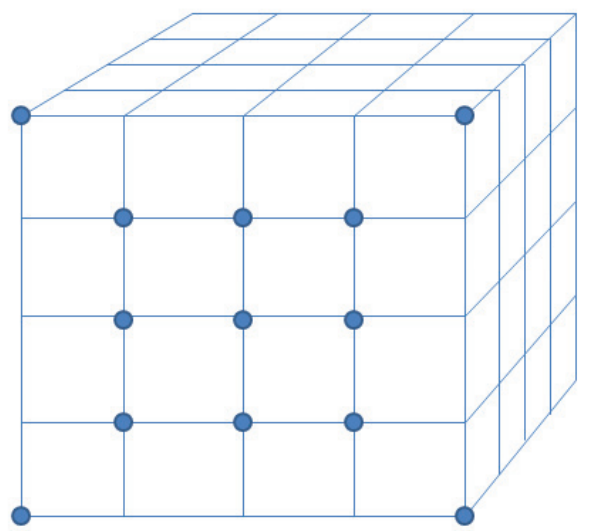

Figure 2. A size 4 cube showing the odd degree vertices on one face of the un-augmented cubic lattice.

In order to solve the problem of odd degree vertices, we have to add augmenting, diagonal edges that turn the corners of the cube into either degree four or degree six vertices, and turn the degree five vertices on the outer faces into vertices of degree either six or eight. We do not have to add augmenting edges to any of the interior vertices since they all have degree six. As we developed design strategies for this structure, we wanted to keep the structure as stable as possible and maintain an easily expandable pattern throughout. To do this, we must add augmenting diagonals to the outside of the cube in a systematic way to preserve the cubes rigidity and symmetry. 


\section{PRELIMINARY OBSERVATIONS}

- There is a difference between cubes of even parity and those of odd parity. Even cubes (size $n$, for $n=2,4,6, \ldots$ ) all have a center vertex in the middle of the structure and in the middle of each face. Odd cubes (size $n$, for $n=1,3,5, \ldots$ ) have a cube in the center of each structure and a square in the middle of each face.

- With this difference between even and odd sized cubes, different design strategies must be considered for each type.

\section{BASIC SIZE 1 CUBIC LATTICE}

We started with a single cube to understand the general nature of cubic structures. A cube is made up of six square faces containing twelve edges and eight vertices each with degree three. However, there is no Euler cycle through the cube due to the odd degree of all of the vertices. Therefore, we have to modify the cube to ensure that all vertices have even degree. This can be achieved by adding a diagonal augmenting edge to each one of the four walls of the cube. With this modification made, we now have eight vertices of degree four and 16 edges. The threading of the cube is depicted in Figure 3 with its respective staples at each vertex. When we discuss a cube, we use the 3D Cartesian coordinate system to locate each vertex in the structure. We place a corner of the cube at the origin, $(0,0,0)$, and extend the rest of the structure along the positive $x, y$ and $z$ axes.

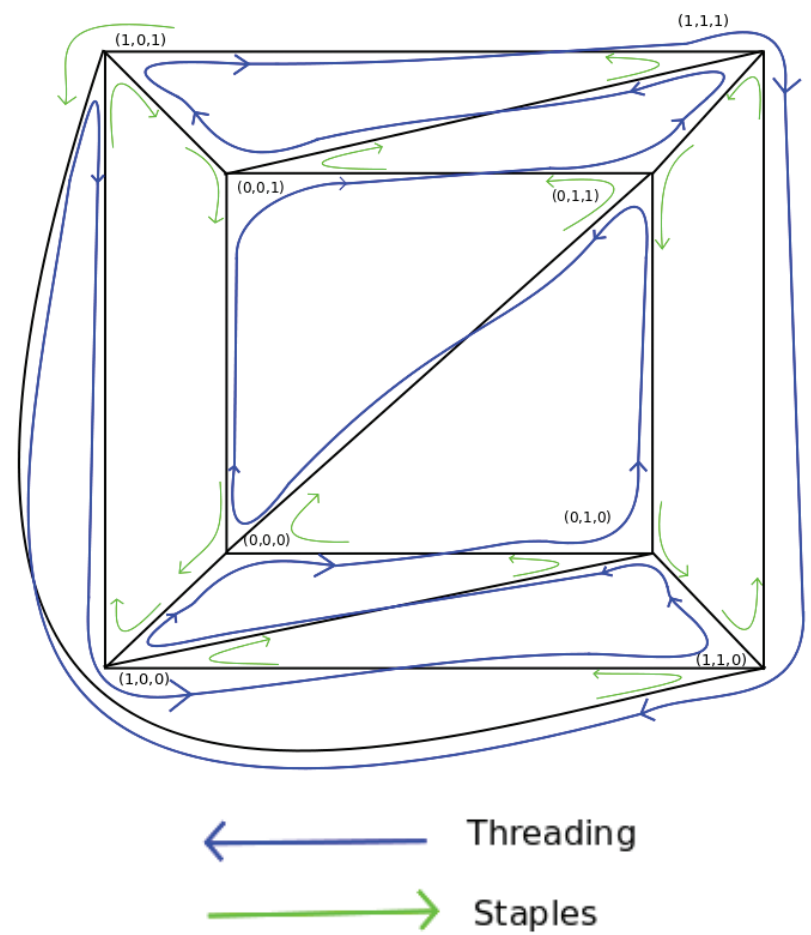

Figure 3. Threading and stapling for a size 1 cube with augmenting edges.

When we consider the threading configuration for each vertex, two different vertex configurations emerge. Vertex configurations that are the same can be rotated to fit the cube as long as the threading and stapling patterns do not change. The difference between the two vertex configurations is in the direction and placement of the threading and staple strands. In the case of the basic size 1 cube, the two different vertex types are mirror images of each other, meaning that both thread 
a $45^{\circ}$ angle and a $90^{\circ}$ angle but travel in opposite directions. This makes them different, yet they still remain symmetric. Figure 4(a) and 4(b) show the two types of vertex configurations that are contained in the size 1 cube along with the coordinates that contain those vertex configurations.

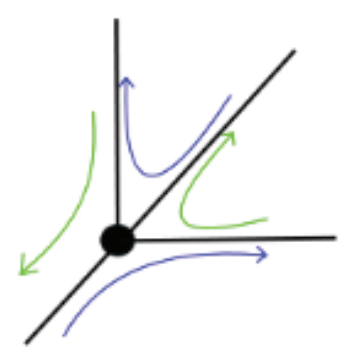

$(0,0,0)$

$(0,0,1)$

$(1,0,0)$

$(1,0,1)$

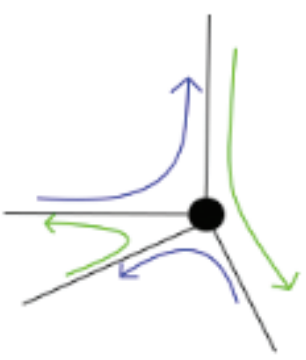

$(0,1,0)$

$(0,1,1)$

$(1,1,0)$

$(1,1,1)$

(a)

(b)

Figure 4. Depicts the two different types of vertex configurations in the size 1 cube.

From this cube we have determined that each corner vertex in a cubic lattice will have to have an odd number of diagonals extending from it to make all corner vertices have even degree. More specifically, there must be one or three diagonals due to the fact that there cannot be more than three diagonals protruding from one corner vertex since all corners of a cube are naturally degree three. Therefore, we cannot have an even number of diagonals coming from any of the corners. 


\section{GENERAL AUGMENTING EDGE DESIGN OF ODD CUBES}

Our designs for each odd size cube, for $n>1$, have a diagonal in each corner of each face. As the cube increases in dimension, more parallel diagonals are added vertically and horizontally from the corner. For example, the size 3 cube has only one diagonal in each corner. The size 5 cube also has a diagonal in each corner plus a parallel diagonal vertically and horizontally adjacent to the corner diagonal. The number of diagonals in each corner continues to grow in this fashion as the cube expands (see Figure 5).

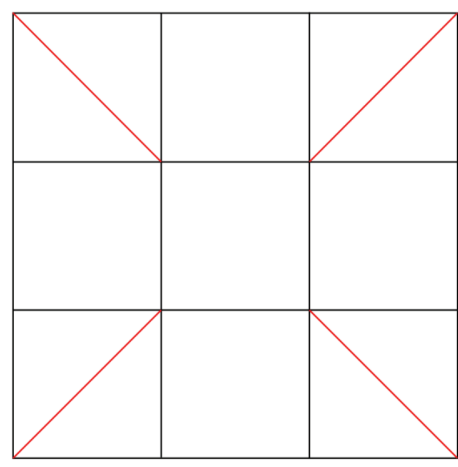

Size 3

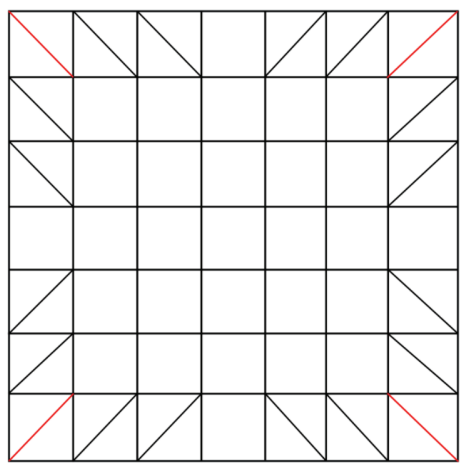

Size 7

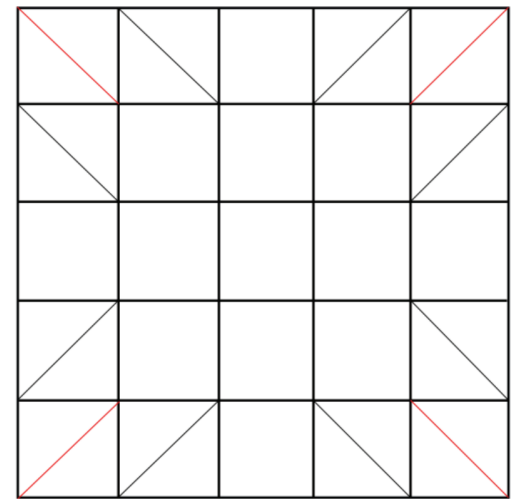

Size 5

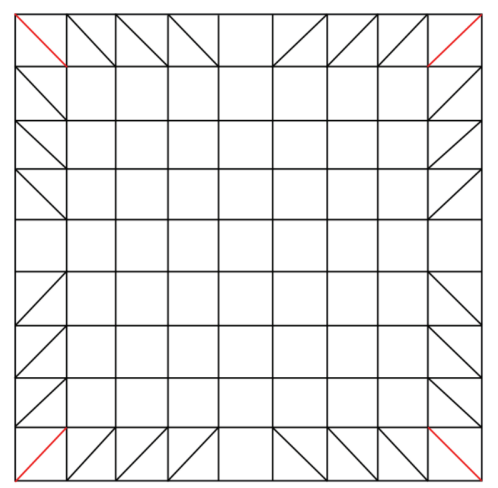

Size 9

Figure 5. Depiction of corner diagonal increasing horizontally and vertically as the odd cube expands.

After we placed these diagonals in each corner of each face we realized that we needed to consider two equivalence classes of odd cubes and use different strategies for the two classes. The congruent modulo $m$ relation $(\mathrm{a} \equiv \mathrm{b} \bmod m$ ), where $m=4$, is the equivalence relation that we used. This means all integers that have the same remainder after division by $\mathrm{m}$ are in the same equivalence class. We found that the odd size cubes, for $n=3,7,11, \ldots$, which are congruent to $3 \bmod 4$, can be constructed in the same manner. Odd size cubes, for $n=5,9,13, \ldots$, which are congruent to $1 \bmod 4$, can be constructed in the same manner (different from the design of odd cubes congruent to $3 \bmod 4$ ). 


\section{DESCRIPTION OF GENERAL DESIGN FOR CUBES CONGRUENT TO 3 MOD 4}

Each face of the odd sized cubes greater than $n=3$ and congruent to $3 \bmod 4(\mathrm{n}=7,11,15, \ldots)$ begins like all odd size cubes, by adding diagonals along edges as shown in Figure 5. Next, we build small candelabras into the center of the face from each corner (see Figure 6). Candelabras should be added to all corners evenly, so if there are 4 candelabras in one corner then all other corners should also have four candelabras. The candelabras create a square in each corner of each face of the cube. This pattern begins as a single candelabra in the size 7 cube, then becomes a $2 \times 2$ arrangement of candelabras in the size 11 cube, a $3 \times 3$ arrangement of candelabras in the size 15 cube and continues in this fashion (see figure 7a.). All problem spots are resolved with these candelabras, but in each face is a cross of empty squares, which could cause the cube to shear. This means that the cube could potentially slide apart and structurally fail. To address this, diagonals are added in a "zigzagging" pattern to wrap around four of the faces of the cube (see figure 7b). When the cube is completed there will be four identical faces which will make up the walls, and the two remaining faces will be identical and form the top and bottom of the cube. The only difference between the four identical faces and the two identical faces is the "zigzag" pattern. (See Appendix for figures of odd designs congruent to $3 \bmod 4$ )

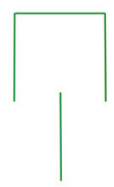

Figure 6. Depiction of small candelabra used in the designs of odd cubes size 5 and greater.
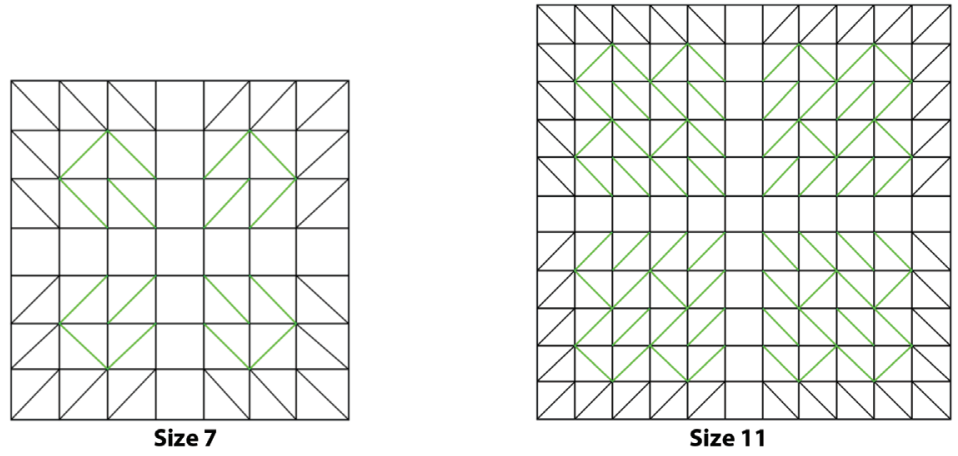

Figure 7a. Depiction of square formed by candelabras in the corner of each face.

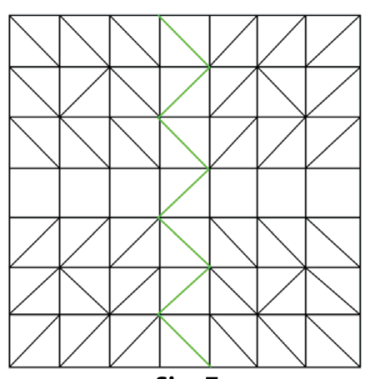

Size 7

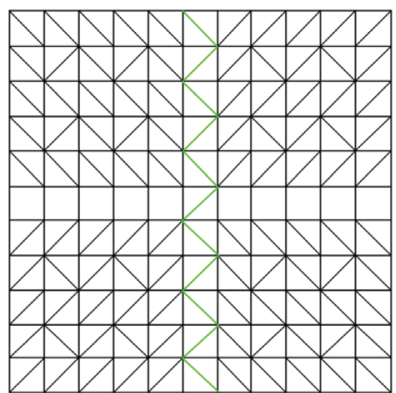

Size 11

Figure 7b. Depiction of "zigzag" pattern that stabilizes the cubes with size congruent to $3 \bmod 4$.

\section{$\mathrm{AJUR}$ Volume 12 | Issue 1 | August 2014}




\section{DESCRIPTION OF GENERAL DESIGN FOR ODD CUBES CONGRUENT TO 1 MOD 4}

Each face of the odd sized cubes congruent to $1 \bmod 4(n=5,9,13 \ldots)$ is created with the same small candelabras (see Figure 6). The design for each face of these cubes begins with the placement of diagonals in the corners and along edges as described above and shown in Figure 5. The candelabras in these cubes are added to each corner in each face in a pinwheel fashion. Instead of creating squares of candelabras in each corner, this design creates rectangles of candelabras that rotate in each corner. The rectangle begins as a $1 \times 2$ arrangement of candelabras in the size 9 cube, then a $2 \times 3$ arrangement in the size 13 cube, a $3 \times 4$ arrangement in the size 17 cube, and continues in this fashion (see Figure 8a). To complete the design, a single candelabrum is placed off of one of the rectangles formed in the corners. When looking at a T-Diagram of the cube (see the Appendix for T-Diagram definition), we have placed this candelabra off of the upper left rectangle of candelabras in each face (see Figure 8b). However, there are multiple ways to place this last candelabrum in each face. The placement we chose is purely for simplicity in constructing the cube. The size 5 cube is too small to include the pin-wheeling candelabras, so each face only contains the diagonals and the one candelabra coming off the upper left corner (see Appendix for Figures of odd designs congruent to $1 \bmod 4)$.
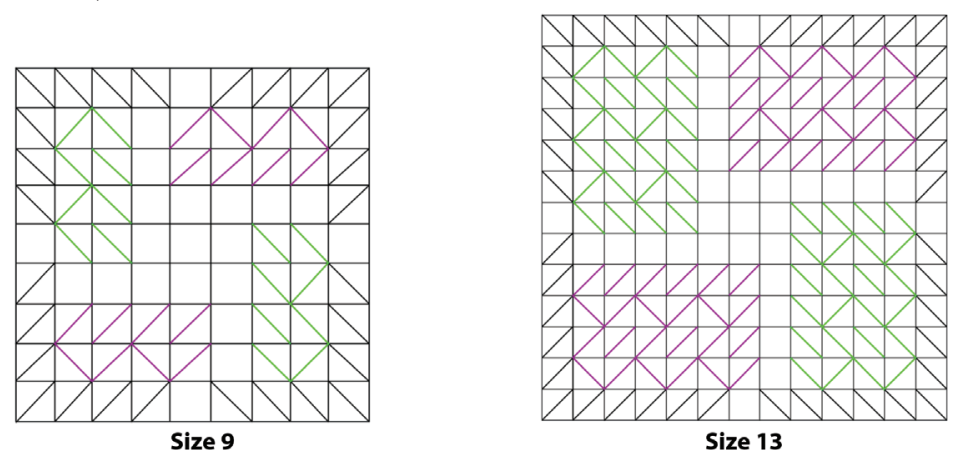

Size 13

Figure 8a. Depiction of pin-wheeling rectangle formed by cubes congruent to $1 \bmod 4$.

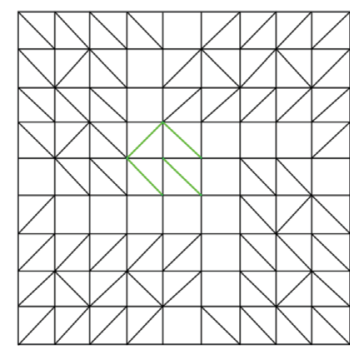

Size 9

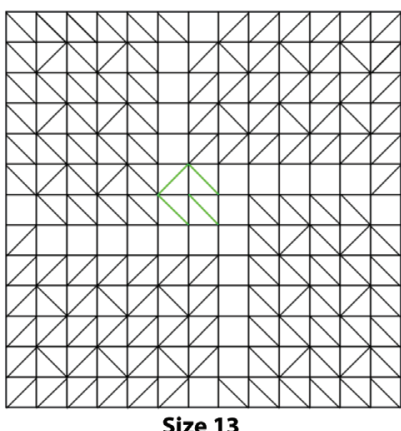

Size 13

Figure $\mathbf{8 b}$. Depiction of center candelabra used in cubes congruent to $1 \bmod 4$. 


\section{SPECIFIC SIZE 3 CUBE DESIGN, THREADING AND STAPLING}

The original size 3 cube contains 64 vertices and 144 edges, and with this original configuration, there are 32 vertices of odd degree. Since this is an odd sized cube, we placed diagonals branching out of each corner of each face. This fixes all 32 of the odd degree vertices by turning the corners from degree three vertices to degree six vertices and turning all of the degree five vertices into vertices of degree six. Since the corner diagonals fix all of our odd degree vertices, we do not need to add any candelabras to the structure. However, since the size 3 cube falls under the category of cubes that are congruent to $3 \bmod 4$, we must place the "zigzagging" pattern through the middle of the cube to prevent it from shearing. The path starts at vertex $(0,0,1)$, travels up to $(0,1,2)$ and then travels down to $(0,2,1)$. The path continues this "zigzag" pattern all the way around the cube and connects with itself at vertex $(0,0,1)$.

The threading of the size 3 cube was done with a "knitting" like approach. The knitting approach of threading slowly traverses the cube by threading sections of the cube at a time. In our case, we threaded the cube column by column (see Figure 9). This style of threading is different than a weaving approach that continuously threads itself from one corner of the cube all the way to the opposite corner and back again. In order for the threading to be possible, we need to ensure that there is an Euler cycle present in the structure that follows the angle constraints of 45 and 90 degrees respectively. Our goal is to create a threading that has symmetry and a fairly simple pattern that can be repeated for other cube sizes.

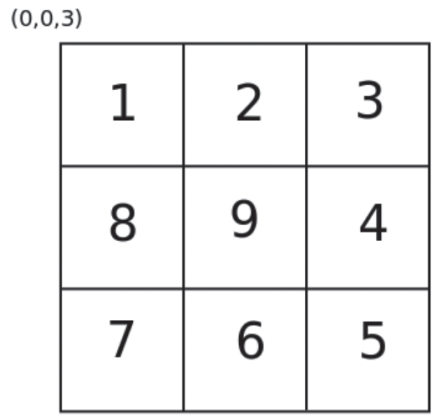

$(3,3,3)$

Figure 9. A labeling of columns in the size 3 cube

The threading starts at $(0,0,0)$ and travels up the first column and down the second, back up the third and down the fourth, etc. (See Appendix for complete threading sequence). Once the cube is completely threaded in this manner, we are able to see that the structure has symmetry in opposite columns. Columns one and five are almost perfectly symmetric (with the exception of one vertex), and columns three and seven are perfectly symmetric. In all, there are 23 different vertex threading configurations in this structure with 64 total vertices (see Figures 10 through 12). 


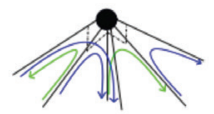

$(0,0,3)$

$(3,3,3)$
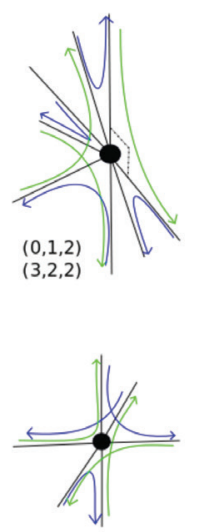

$(1,1,2)$
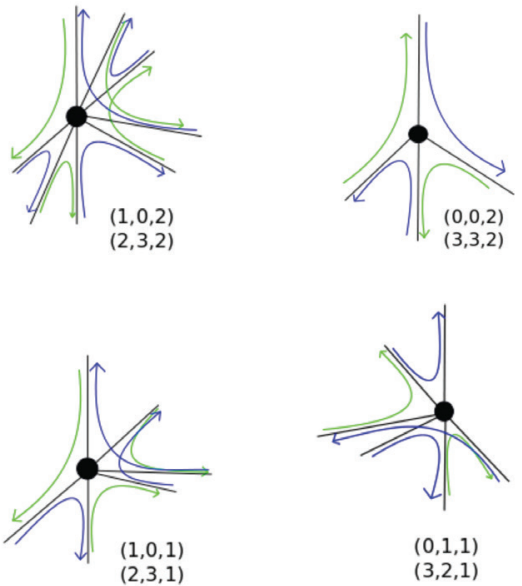

Figure 10. Vertices exclusively contained in columns 1 and 5 ,

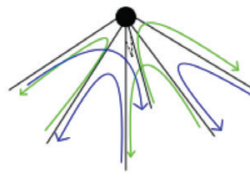

$(0,3,3)$

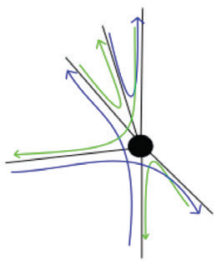

$(1,3,2)$
$(2,0,2)$

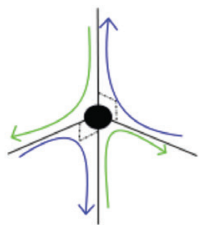

$(0,3,1)$

$(3,0,1)$

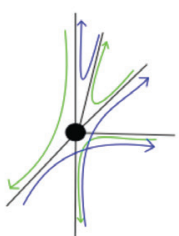

$(0,2,2)$

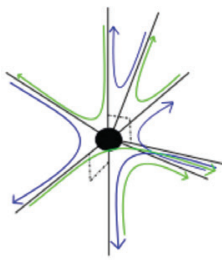

$(0,2,1)$

$(3,1,1)$

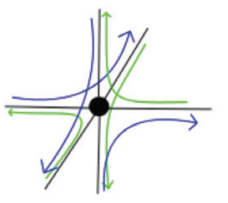

$(1,2,2)$

$(2,1,2)$

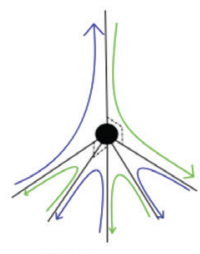

$(3,0,2)$
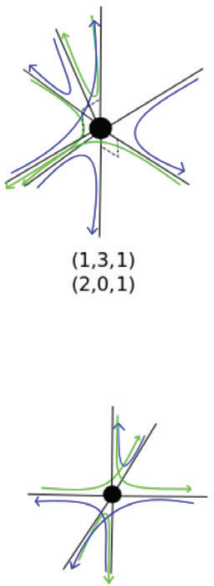

$(1,2,1)$

Figure 11. Vertices exclusively contained in columns 3 \& 7 . 

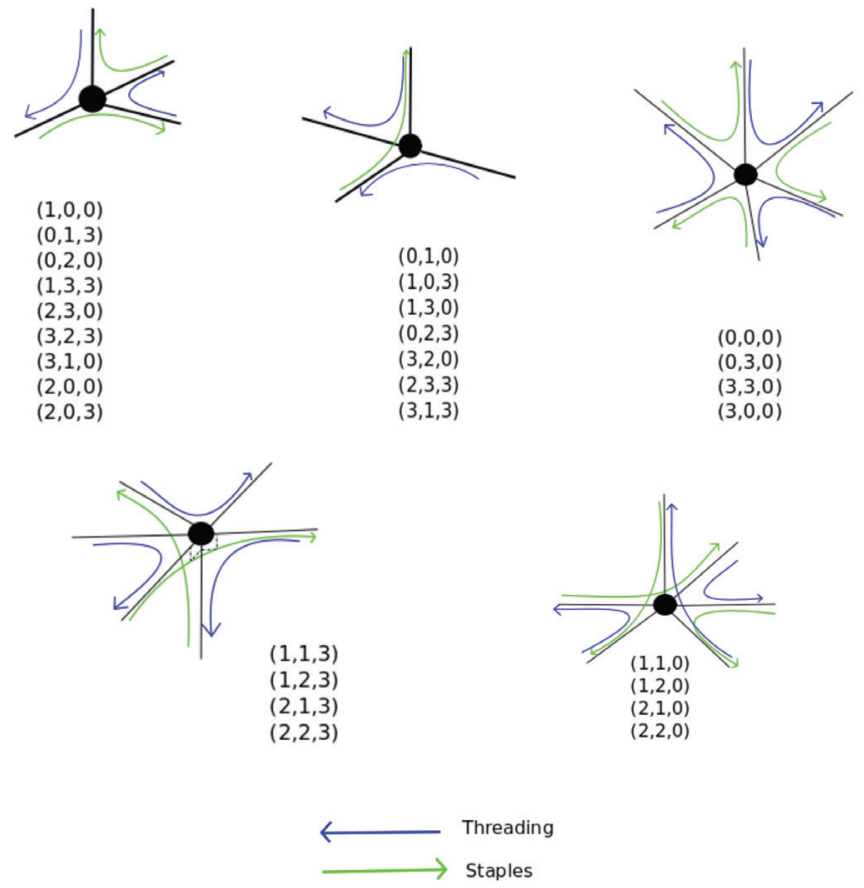

Figure 12. Vertices that are contained in all four columns $1,3,5$ \& 7

\section{GENERAL AUGMENTING EDGE DESIGN OF EVEN CUBES}

Unlike the odd sized cubes which have a square in the middle of each face and a cube at the center of its structure, the even sized cubes $(n=2,4,6 \ldots)$, as discussed in the preliminary observations, have a vertex in the center of each face and in the very center of the entire structure. This makes a difference in approaching possible designs, because there are an odd number of vertices inside each face of the even cube and an even number of vertices per face for odd cubes. The odd number of vertices in each face of an even sized cube makes it impossible to pair all vertices in one face. However, since there is an even number of vertices on the surface of the entire cube, we can pair an odd degree vertex on one face with an odd degree vertex on an adjacent face and make them even. Our general even design is created by building large candelabras into the center from two opposite corners (see Figure 13a). These large candelabras are built in towards the middle until the ends of the candelabras from opposite corners connect. At the vertex where the ends of the candelabras connect, a diagonal is added to create a "T" (see Figure 13b).We omit an edge from one candelabra in order to create one odd degree vertex that can then pair with the odd degree vertex on an adjacent face (see Figure 14 for construction of the even cube face). The design of one face of the cube can be rotated in different directions to create the faces of the entire cube. The completed cube will have three pairs of identical faces (see Figure 15 for the proper rotation of faces).

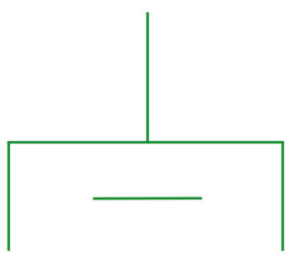

(a)

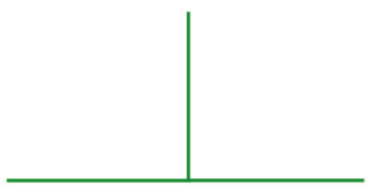

(b)

Figure 13. (a) depicts the large candelabra used in the even cube design, and (b) shows the " $\mathrm{T}$ " that is created after the opposite candelabras connect. 


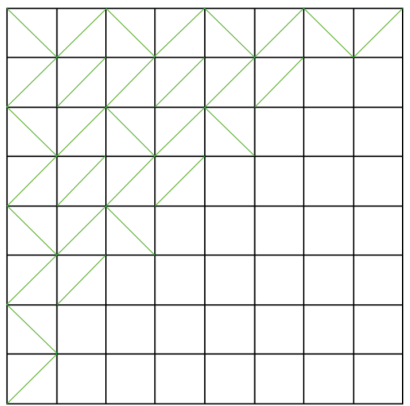

(a)

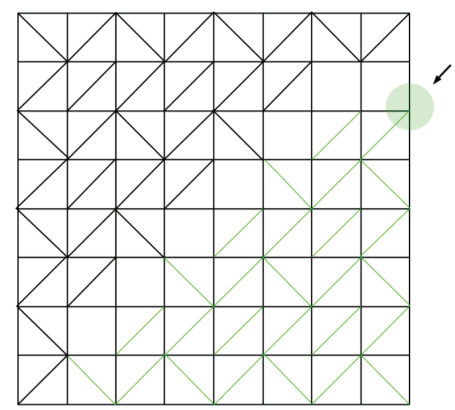

(b)

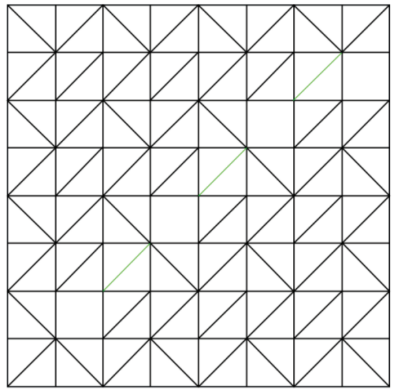

(c)

Figure 14. (a) depicts the construction of large candelabras from one corner of a face for a size 8 cube. (b) Shows the construction of candelabras starting from the opposite corner leaving one unconnected candelabra that connects with its adjacent face. (c) Depicts the additional diagonals that form the "T" shape discussed above.

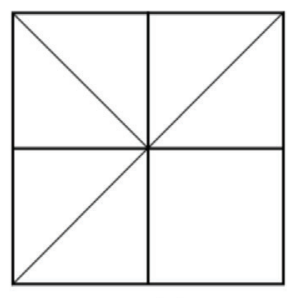

(a)

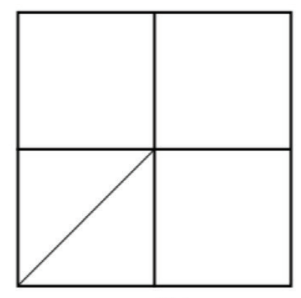

(b)

Figure 15. Depicts the rotation of even cube faces. 


\section{SPECIFIC SIZE 2 CUBE DESIGN, THREADING AND STAPLING}

The original size 2 cube consists of 27 vertices and 54 edges, and out of these 27 vertices, 12 of them are of odd degree. To make these vertices even degree, augmenting edges are added to make a total of 66 edges. Three diagonals are placed coming out of two corner vertices that are opposite of each other, $(0,0,0)$ and $(2,2,2)$. The rest of the corner vertices only have one diagonal coming out of them. The diagonals should be placed so that the completed cube has two different types of faces (see Figure 16). One type of face contains three diagonals that form the " $\mathrm{T}$ " shape used in all even cubes, and the other contains only one diagonal protruding from the corner vertex. These two types can be rotated to create all other faces of the cube (see Appendix for T-diagram). Now, all vertices become a degree 4,6 , or 8 , making it possible to thread the cube.

The threading of the size 2 is done in the same knitting like pattern as the size 3 cube and traverses the cube column by column in a similar manner. However, since the cube design itself has less than ideal symmetry, the threading sequence also has less symmetry. Opposite corners, $(0,0,0)$ and $(2,2,2)$, are purposely threaded exactly the same and the rest of the cube's threading follows from that. The final threading for the size 2 cube has 16 different vertex configurations for 27 vertices (see Figure 17).

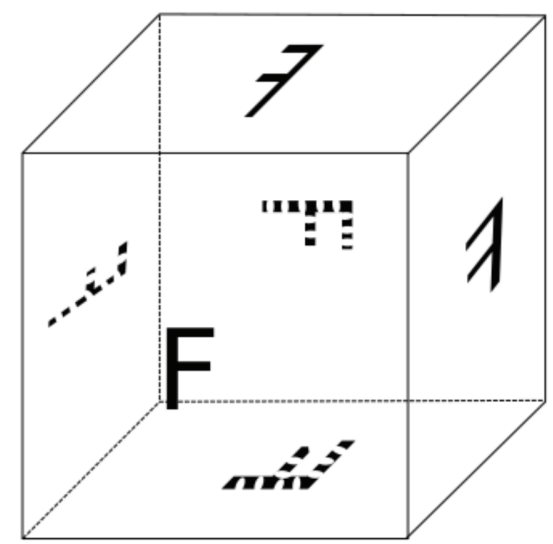

Figure 16. (a) and (b) depict the two types of faces used to construct the size 2 cube. 

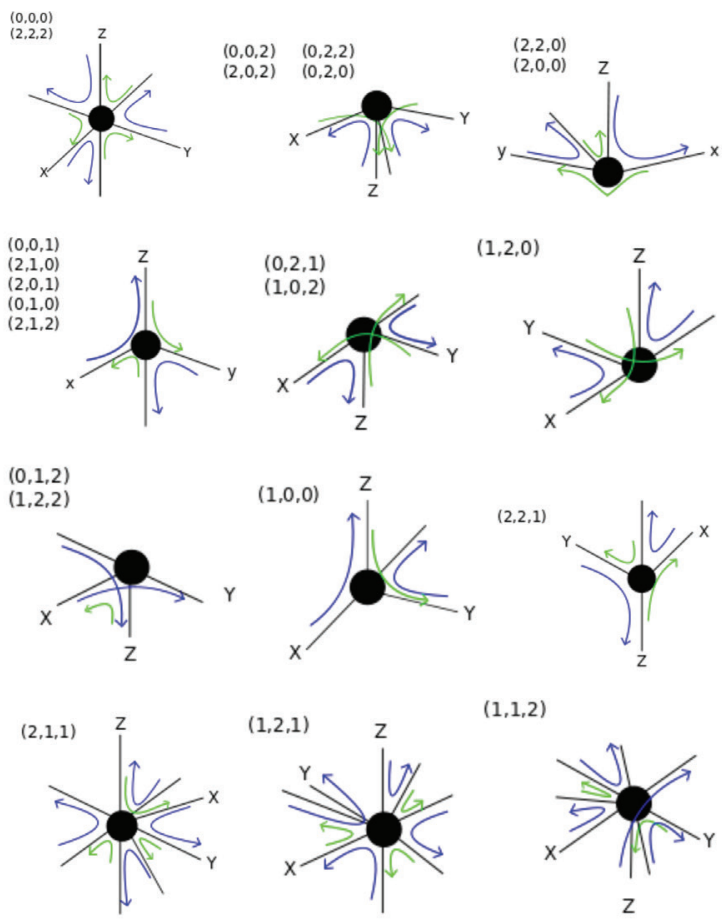

$(1,1,2)$
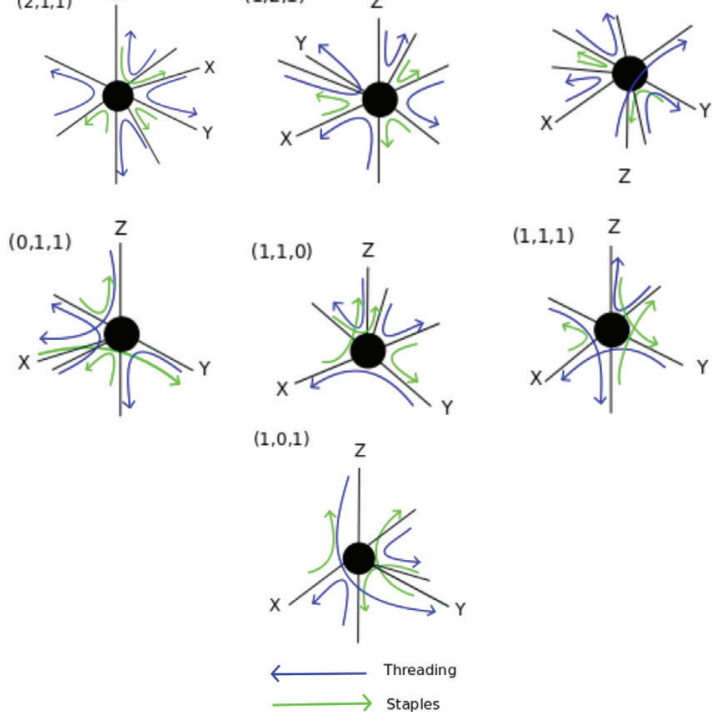

Figure 17. Depicts all vertex configurations in the threading and stapling of the size 2 cube.

\section{CONCLUSION}

Through our work with the cubic lattice, we have developed designs that ensure an Euler cycle exists in all of the cubic structures. The designs that have been created are specific to the parity of the cube, odd or even. In the case of the odd cubes, there are two types of equivalence classes that exist, cubes congruent to $1 \bmod 4$ and cubes congruent to $3 \bmod 4$. All of these designs contain relative symmetry and are able to expand. In the specific cases of size 1, 2 and 3 cubes, we provided threading configurations and techniques that can be applied to the construction of these cubes. These threading techniques seem to work well for the cubic lattice; however we wish to further develop a more general threading technique that can be applied to all cubes in the future.

\section{AJUR volume 12 | Issue 1 | August 2014}




\section{ACKNOWLEDGEMENTS}

We thank Ned Seeman of Seeman's lab at NYU for helping us develop our design constraints and the National Science Foundation (NSF) grant number 10-GR150-514030-11 for their funding of this project. We also thank our research advisors, Joanna Ellis-Monaghan and Greta Pangborn.

\section{SUPPLEMENTAL INFORMATION}

Supplemental appendices may be found online in PDF format alongside the associated article at www.ajuronline.org.

\section{REFERENCES}

[1] Zadegan, R. M., and Norton, M. L. (2012) Structural DNA nanotechnology: from design to applications, International journal of molecular sciences 13, 7149-7162.

[2] Chen, J. H., and Seeman, N. C. (1991) Synthesis from DNA of a molecule with the connectivity of a cube, Nature 350, 631-633.

[3] Zhang, Y., and Seeman, N. C. (1994) Construction of a DNA-truncated octahedron, Journal of American Chemistry 116, 1661-1669.

[4] Shih, W. M., Quispe, J. D., and Joyce, G. F. (2004) A 1.7-kilobase single-stranded DNA that folds into a nanoscale octahedron, Nature 427, 618-621.

[5] He, Y., Ye, T., Su, M., Zhang, C., Ribbe, A. E., Jiang, W., and Mao, C. (2008) Hierarchical self-assembly of DNA into symmetric supramolecular polyhedra, Nature 452, 198-201.

[6] Girard, J., Gilbert, A., Lewis, D., and Spuches, M. (2011) Design Optimization for DNA Nanostructures, American Journal of Undergraduate Research 10.

[7] Tucker, A. (2007) Applied Combinatorics, John Wiley \& Sons, Inc., New Jersey.

\section{ABOUT THE STUDENT AUTHORS}

Miranda LaRocque graduated from Saint Michael's College with a BS degree in Mathematics in May 2014. She is currently working as an Actuarial Systems Analyst at National Life Group, a life insurance company in Montpelier, Vermont.

Tyler Hotte graduated from the Saint Michael's College and the University of Vermont in May 2014 with a BS degree in Mechanical Engineering (minor in math). He is currently living in Burlington, VT and works as a Timber Framer for Vermont Frames in Starksboro, VT.

\section{PRESS SUMMARY}

DNA nanostructures are nanoscale structures made of self-assembling DNA, which can be used for biomolecular computing, targeted drug delivery, and biosensors. We use the Watson-Crick properties of DNA and the principles of graph theory to construct origami folding designs for a cubic lattice. Our methods involve a single scaffolding strand of DNA and short staple strands of DNA that secure the scaffolding strand into the shape of a cubic lattice. Our objective was a mathematical design strategy that could be expanded systematically for self-assembly of any size cubic lattice. 


\title{
A Student Approach to a Mathematical Simulation of a Racing Electric Vehicle
}

\author{
Fernando Daniel León-Cázares and Daniel Xoconostle-Luna* \\ Instituto Tecnológico y de Estudios Superiores de Monterrey Campus Estado de México \\ *Students: fernando.leon.cazares@gmail.com,dxoconos@hotmail.com \\ Mentor: jorge.mendoza@itesm.mx
}

\begin{abstract}
A program was developed using the software Mathematica to simulate the dynamical behavior of an electric racing car, an electrathon. In conjunction with experimental data it is focused to allow the Borregos-CEM Racing Team to decide which settings have to be adjusted in order to increase the velocity of the racing car while decreasing its energy consumption, i.e. the current demanded to the batteries.
\end{abstract}

\section{KEYWORDS}

model, electric, racing, vehicle, dynamic, simulation, electrathon

\section{INTRODUCTION}

Electrathon began in England in 1978. Then it spread to Australia in 1980, the U.S.A. in 1990, and to Mexico in $1993 .^{1}$ After two years in Mexico it became an open championship to all universities and private participants. As of 2012, the championship involved 38 cars that ran with DC motors and commercial lead-acid batteries; the seven races were contested in several states across the Republic. While the future waits with great promises of electric and battery technologies, in the Borregos-CEM Racing Team, we are producing an EV (Electric Vehicle) beyond the championship's expectations. (From this point forwards, Team is an abbreviation of Borregos-CEM Racing Team)

To construct a car that rolls is quite easy, but to build a proper Electrathon there has to be an active awareness of the many details that are a natural part of the car. Then, to fashion the best possible racing EV there ought to be a CAD (Computer Aided Design), a CAE (Computer Aided Engineering) and, in the Team's case, the creation of a mathematical model of the car dynamics that is understandable to all.

Such a computer program was developed using the software Mathematica, to simulate the dynamical behavior of an Electrathon, which raced with DC (Direct Current) until 2012. Since the races last 90 minutes and allow a change of batteries, the championship focus is the optimization of the energy usage and limiting weight of the batteries. Hence, efficiency, defined as the ratio between the distance traveled and the electric charge consumed, remains as the most important factor to optimize. Thus, the whole idea of elaborating a theoretical model is to enhance the car's performance in the championship, which means an improvement in energy consumption. Because of this, the model is by now in its first steps to be a complete platform that includes all the dynamical properties of an EV, but that is flexible enough to be modified so that it successfully simulates a gasoline car.

The theoretical model in which the software is based was developed in order to obtain the required equations. This considers mechanical and electrical parameters that affect the vehicle's performance and may be optimized so that its velocity increases while its consumed charge decreases. Afterwards, it is explained how experimental data was obtained and compared with the simulations to validate the theoretical model previously mentioned. 


\section{THEORETICAL MODEL}

First of all a briefly explanation of the car to which the program was developed is given. Its powertrain consists of an electric DC motor, a roller chain, two sprockets, the rear shaft and both rear wheels. There is also a device ${ }^{2}$ that enables the slower rear wheel to lose traction in a curve so that the car can turn properly, as well as to completely avoid the power transmission when the angular velocity of the wheels is higher than the one delivered by the shaft.

The electric system has an electronic adjustable controller that allows the user to set a maximum output current as a percentage of the demanded by the motor in normal conditions. Once the motor curves of torque and current versus angular velocity are interpolated into the program, it is possible to obtain the force applied to the vehicle $F_{m}$ as

$$
F_{m}(v)=\frac{c l}{s r_{w}} * T_{m}\left(\frac{30 v}{\pi s r_{w}}\right),
$$

Equation 1.

where $c l$ is the controller limit (it can vary from 0 to 1 ), $s$ is the sprocket ratio of speed amplification, $r_{w}$ the wheel radius and $v$ the linear velocity of the vehicle. The function $T_{m}(\eta)$ is the interpolation of the motor's curve of torque versus angular velocity $\eta$ (in $\mathrm{N} \cdot \mathrm{m}$ and rpm respectively). Equation (1) is obtained by transmitting the motor's torque output to a linear force acting directly on the car, as well as by developing the necessary dimensional analysis to express all the parameters using the International System of Units.

Even though the mass of the vehicle remains unchanged during the competition it is a really important parameter. Also, its geometry should be taken into account due to the effect of the drag force $F_{d},{ }^{3}$ which can be approximated for a vehicle as

$$
F_{d}(v)=\frac{1}{2} C_{d} \rho A v^{2},
$$

Equation 2.

where $C_{d}$ is the drag coefficient, $\rho$ the air's density (considered as a constant equal to $\left.1.2041 \mathrm{~kg} / \mathrm{m}^{3}\right), v$ the linear velocity of the car, and $A$ its frontal area. Considering a parameter $\beta=C_{d}$ $A$, equation (2) becomes

$$
F_{d}(v)=0.60205 \beta v^{2} .
$$

Equation 3.

Friction due to mechanical interactions between the components of the vehicle represents also a force that acts against motion. However, it is neglected as it is really small when compared with the drag force. ${ }^{4}$

The last force taken into account is the one produced by gravity, $F_{g}$, when the slope is other than zero. It can be expressed as

$$
F_{g}=m g \sin (-\alpha),
$$

Equation 4.

where $m$ is the vehicle's mass and $\alpha$ the angle between a horizontal plane and the one with the slope (an angle $\alpha>0$ means that the car is going up).

Given the 2nd Newton's Law, the car's acceleration $a(v)$ can be calculated as

$$
a(v)=\frac{F_{m}(v)-F_{d}(v)+F_{g}}{m},
$$

Equation 5.

and substituting equations (1), (3) and (4) into (5), as well as considering that $a(v)=d v / d t$, we get the ordinary differential equation

$$
v^{\prime}(t)=\frac{c l}{s m r_{w}} * T_{m}\left(\frac{30 v(t)}{\pi s r_{w}}\right)-\frac{0.60205 \beta v(t)^{2}}{m}+g \sin (-\alpha), \quad \text { Equation } 6 .
$$


with the initial velocity $v_{0}$ known

$$
v(0)=v_{0},
$$

Equation 7.

in terms of $v(t)$, where $t$ is the time. This can be solved numerically in Mathematica no matter what form has the motor's curve. Given the function $v(t)$ it is possible to find the displacement $x$ traversed by the vehicle as a function of time as

$$
x(t)=\int_{0}^{t} v(t) d t
$$

Equation 8.

The total time $t_{f}$ necessary for the car to complete a straight segment of a circuit can be calculated by solving for the time the equation

$$
x(t)=\frac{x_{f}}{\cos (\alpha)},
$$

Equation 9.

where $x_{f}$ is the horizontal length of that segment. It is important to note that for a circular path it is necessary to change this length $x_{f}$ for $r^{*} \theta$, where $r$ is its radius and $\theta$ its angle of displacement. Just for this case it is also possible to obtain a function of the centripetal acceleration $a_{c}$ in terms of time as

$$
a_{c}(t)=\frac{v(t)^{2}}{r} .
$$

\section{Equation 10.}

After this analysis it is possible to determine the dynamical behavior of an accelerating electric vehicle. Nevertheless, it is also an objective of this program to obtain a function of the charge demanded to the batteries. In order to accomplish this, it is necessary to interpolate a function of the current $I_{m}\left(T_{m}(\eta)\right)$ in terms of the motor's torque. This information is given by its manufacturer. Taking the controller into account, as well as the relationship between the vehicle's angular and linear velocity, a function in terms of the last one, $I_{l v}$, can be stated as

$$
I_{l v}(v)=c l * I_{m}\left(T_{m}\left(\frac{30 v}{\pi s r_{w}}\right)\right)
$$

Equation 11.

To get the instantaneous current at any time it is just required to evaluate in a function $I(t)$ in terms of the time as

$$
I(t)=I_{l v}(v(t)),
$$

Equation 12.

and finally the total charge $Q$ consumed in a segment of a circuit is obtained as

$$
Q=\int_{0}^{t_{f}} I(t) d t
$$

Equation 13.

Given all these equations, a complete circuit can be run, taking into account that the initial velocity of each segment is the same as the one obtained at the last instant of the previous one. After adding each time and charge consumed, many laps can be rapidly simulated. It is also possible to develop the model with no torque from the motor, so that there are segments in which the pilot does not accelerate. 


\section{EXPERIMENTAL}

The motor used was a Briggs and Stratton Etek ${ }^{5}$ at $36 \mathrm{~V}$. However, as it may be seen, the manufacturer's data sheet only shows the information for the range from nearly 2125 to $2592 \mathrm{rpm}$. Also, a maximum torque of $47.73 \mathrm{~N} \cdot \mathrm{m}$ is reported, as well as a linear relationship between this property and the current demanded of $0.1288 \mathrm{~N} \cdot \mathrm{m} / \mathrm{A}$. The controller used was an Alltrax Axe $48455^{6}$ For the simulations, a linear interpolation for the unknown range of angular velocities was used. The vehicle, including the batteries and the pilot, had a mass of $192 \mathrm{~kg}$, its wheels had a 5 inch radius and it was possible to estimate the coefficient $\beta$ with the help of the software Ansys Fluent running a simulation with the vehicle's CAD. This simulation's output showed that the car had a drag coefficient of 0.24018 and an estimation of a $1.444 \mathrm{~m}^{2}$ frontal area; of which $0.622 \mathrm{~m}^{2}$ correspond to the bodywork. However, the remaining exposed area has a higher drag coefficient. Hence the calculated $\beta=0.346$ for the bodywork had to be increased to include the rougher surface of the rest of the car. Several simulations were run to fit this parameter with the results of a previous race. As it can be seen in Figure 1, a coefficient $\beta=0.8 \mathrm{~m}^{2}$ proved to be the best fit, so that further simulations were closer and more accurate.

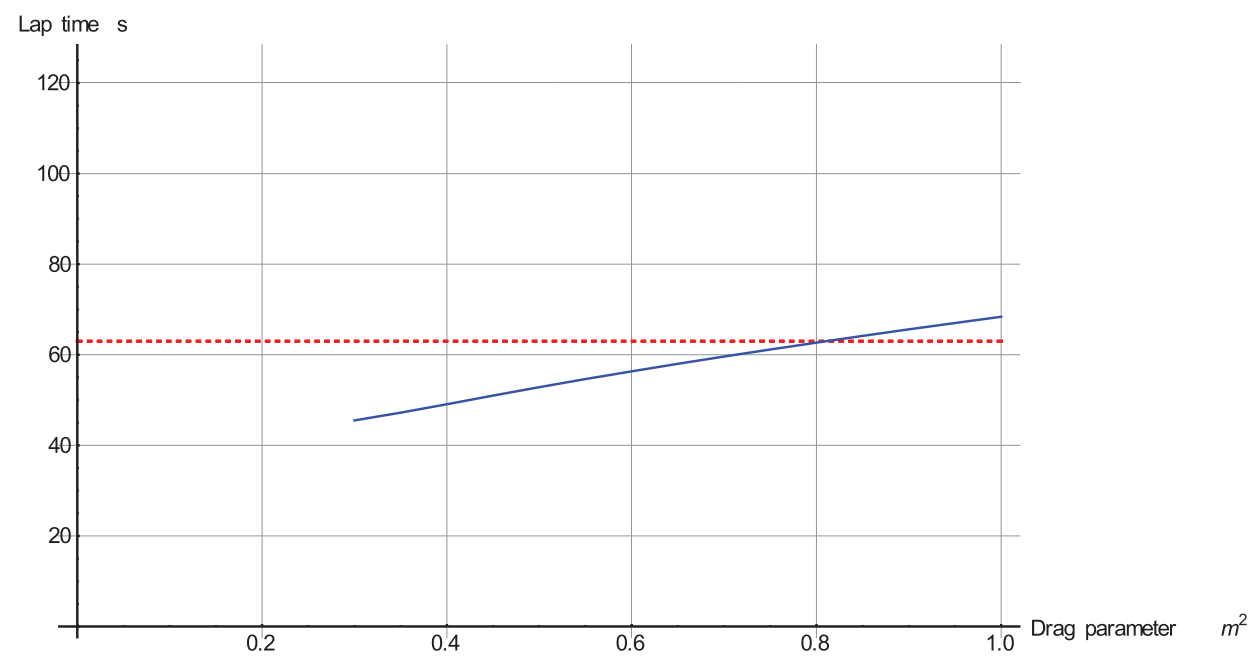

Figure 1. Experimental fit of the drag parameter $\beta$ from a previous race in Kartódromo La Mina, Toluca, Mexico at August 26th, 2012 for the average lap time (dashed line) of $63.03 \mathrm{~s}$. The solid line represents the simulations run by the software for different values of $\beta$.

To further validate the program it was necessary to gather as many reliable experimental data as possible. Measures were taken from two sources: straight line sprints and during the races. For the sprints, the car was equipped as it would be for any race and a set distance is measured from 0 to 200 meters with marks every 50 meters. The test began with a pre-set sprocket ratio of 0.5357 and the controller limit was changed after each sprint to $0.2,0.3,0.5$ and 0.7 . Afterwards, the same values were tested for a ratio of 0.4643 . This test was designed to run the car in a controlled environment (climate conditions may vary even during a test) and to know how these parameters affect its velocity.

In the championship, it was possible to base the decision of the parameters' values to use just in the last race. Each circuit was modeled by segmenting it in many parts, all of them straight lines or perfect arcs of a circle, and measuring them with the help of Google Earth. This software was also helpful for determining the angles of each division's different slopes as it offers a complete height profile of the terrain. However, even though it was difficult to take a continuous measurement of the velocities and currents, we managed to take the times of each lap to compare them with the 
expected ones. There are many factors, such as the pilot and the other contestants, which make it complicated to quantitatively obtain a constant performance. Even when the manufacturers give the theoretical total charges of the batteries, ${ }^{7}$ it varies depending on the discharge speed ${ }^{8}$ and their voltage drop makes it difficult to determine when they can be considered as depleted. Also, as the voltage decreases, the overall performance of the car gets continuously modified. This is why we could only appreciate a qualitative change with respect to the previous races.

\section{RESULTS}

To validate the accuracy of the software model, we used the gathered data of the tests (Table 1) to compare it against the results that the program was predicting (Table 2).

\begin{tabular}{|c|c|c|c|c|c|c|c|c|}
\hline \multicolumn{9}{|c|}{ Experimental data } \\
\hline \multirow{2}{*}{$\begin{array}{l}\text { Length } \\
{[\mathrm{m}]}\end{array}$} & \multicolumn{8}{|c|}{ Time $[s]$} \\
\hline & $\begin{array}{l}\mathrm{s}=0.5357 \\
\mathrm{cl}=0.2\end{array}$ & $\begin{array}{l}\mathrm{s}=0.5357 \\
\mathrm{cl}=0.3\end{array}$ & $\begin{array}{l}\mathrm{s}=0.5357 \\
\mathrm{cl}=0.5\end{array}$ & $\begin{array}{l}\mathrm{s}=0.5357 \\
\mathrm{cl}=0.7\end{array}$ & $\begin{array}{l}s=0.4643 \\
c l=0.2\end{array}$ & $\begin{array}{l}s=0.4643 \\
c l=0.3\end{array}$ & $\begin{array}{l}\mathrm{s}=0.4643 \\
\mathrm{cl}=0.5\end{array}$ & $\begin{array}{l}\mathrm{s}=0.4643 \\
\mathrm{cl}=0.7\end{array}$ \\
\hline 50 & 12.4 & 10.3 & 7.6 & 6.7 & 11.8 & 9.2 & 6.9 & 6.7 \\
\hline 100 & 19 & 15.3 & 11 & 10 & 17.5 & 13.6 & 10.5 & 10 \\
\hline 150 & 23.9 & 19.1 & 14.2 & 13.1 & 21.8 & 16.8 & 13.8 & 13.5 \\
\hline 200 & 27.1 & 21.6 & 16.6 & 15.9 & 25.6 & 20.3 & 17.1 & 16.8 \\
\hline
\end{tabular}

Table 1. Experimental data of the sprints for length intervals of $50 \mathrm{~m} . s$ is the sprocket ratio of speed amplification and $c l$ the controller limit.

\begin{tabular}{|c|c|c|c|c|c|c|c|c|}
\hline \multicolumn{9}{|c|}{ Simulation data } \\
\hline \multirow{2}{*}{$\begin{array}{l}\text { Length } \\
{[\mathrm{m}]}\end{array}$} & \multicolumn{8}{|c|}{ Time $[\mathrm{s}]$} \\
\hline & $\begin{array}{l}\mathrm{s}=0.5357 \\
\mathrm{cl}=0.2\end{array}$ & $\begin{array}{l}\mathrm{s}=0.5357 \\
\mathrm{cl}=0.3\end{array}$ & $\begin{array}{l}\mathrm{s}=0.5357 \\
\mathrm{cl}=0.5\end{array}$ & $\begin{array}{l}\mathrm{s}=0.5357 \\
\mathrm{cl}=0.7\end{array}$ & $\begin{array}{l}\mathrm{s}=0.4643 \\
\mathrm{cl}=0.2\end{array}$ & $\begin{array}{l}\mathrm{s}=0.4643 \\
\mathrm{cl}=0.3\end{array}$ & $\begin{array}{l}\mathrm{s}=0.4643 \\
\mathrm{cl}=0.5\end{array}$ & $\begin{array}{l}\mathrm{s}=0.4643 \\
\mathrm{cl}=0.7\end{array}$ \\
\hline 50 & 12.628 & 10.444 & 8.259 & 7.101 & 11.918 & 9.89 & 7.864 & 6.792 \\
\hline 100 & 18.673 & 15.533 & 12.4 & 10.745 & 17.732 & 14.823 & 11.929 & 10.45 \\
\hline 150 & 23.779 & 19.87 & 15.98 & 13.952 & 22.69 & 19.077 & 15.548 & 13.88 \\
\hline 200 & 28.459 & 23.872 & 19.318 & 17.042 & 27.267 & 23.036 & 19.066 & 17.276 \\
\hline
\end{tabular}

Table 2. Simulated data of the sprints for length intervals of $50 \mathrm{~m} . s$ is the sprocket ratio of speed amplification and $c l$ the controller limit.

The average error obtained from comparing tables 1 and 2 was about $6.85 \%$. This is relatively low, and it may occur due to the lack of the motor's information at low speeds, as well as to measurement errors, battery discharging from one test to another, wind effects and different tire temperatures, among others.

The race took place in a parking lot at ITESM Guadalajara and the average lap time was of 44 seconds. The sprocket ratio used was of 41.67 , while the controller limit was set to $25 \%$. On the other hand, the simulation output for 4 laps was the following one:

Lap 1: Time $=51.6364 \mathrm{~s}$; charge $=2006.55 \mathrm{C}$

Lap 2: Time $=43.5078 \mathrm{~s}$; charge $=1234.42 \mathrm{C}$

Lap 3: Time $=43.5044 \mathrm{~s}$; charge $=1233.78 \mathrm{C}$

Lap 4: Time $=43.5044 \mathrm{~s}$; charge $=1233.78 \mathrm{C}$ 
As it may be seen, the time per lap reaches a minimum after several iterations, that fact that does not apply for real life. The simulation error obtained was of about $1.126 \%$ in comparison with the measured times. Figure 2 shows a graph of velocity versus time, where it is possible to appreciate how terrain elevations and segments in which the car is not allowed to accelerate make the profile obtained a variable one.

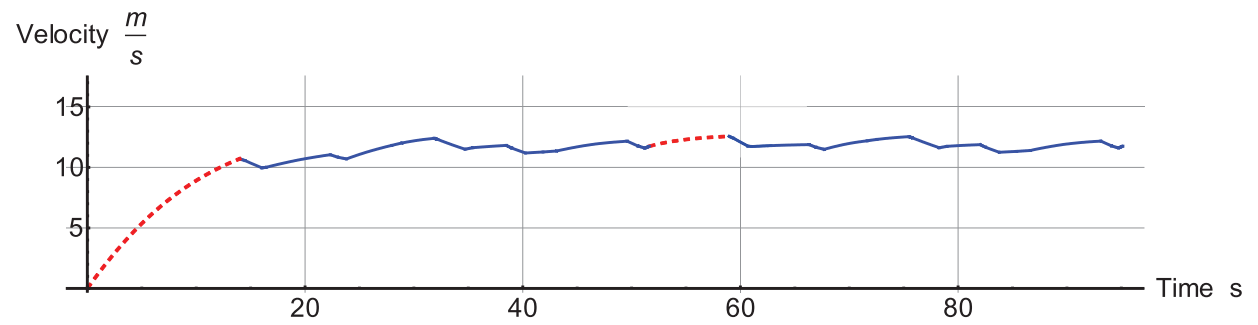

Figure 2. Simulation output for the first two laps of the championship's race in Instituto Tecnológico y de Estudios Superiores de Monterrey Campus Guadalajara at November 3rd, 2012. Dashed lines represent the first segment of each lap.

Regarding the electric performance, we were unable to accurately measure the current at each point of the circuit. However, we could ensure that the batteries never reached a critical point and the car maintained a regular time in each lap.

This means that the developed program is useful to predict the results with ever increasing accuracy, as long as the feedback is fresh data. A major point of the program is that by running a large number of simulations, with different controller limits and sprocket ratios, a decision matrix can be made so that the Team can decide which values is the optimum to use in each race. Even though physical testing was sought every day, due to the time required to charge the batteries and the risk of injuring the vehicle, the simulations provided the best source of information we could acquire while not in a race.

\section{CONCLUSIONS}

This present model, as shown in the results, has proved to be a useful tool to optimize some parameters of the vehicle and to show how each of them affect its dynamical and electrical behavior. However, there is some variation between the real performance and the one predicted by the simulations due to many factors. Even though the lack of information regarding the motor curves and possible variations from its theoretical operation represent a serious inconvenience, it is possible to circumvent this issue by adequately testing the used motor. Regarding the $\beta$ coefficient, it was possible to fit this value to a real approximation by using data from previous races. With the necessary experimentation and simulations it is possible to find a more accurate value of this parameter. Variable battery voltages during the races due to discharge make it impossible to have a constant behavior, resulting in changes from the simulation outputs. Nevertheless, it is possible to neglect these variations as the voltage drop becomes important only after long periods of time.

Even while this program is still being improved, it is already offering good results the Team can exploit. As it is shown in the results, this software can be really useful to predict the behavior of the car and to determine the parameters to be used in every race, taking into account the terrain elevation profile. By this it is possible to adjust the simulation to the given conditions in each race. The obtained results in the sprint tests, in conjunction with the ones in the circuits, proved to have a percentage error of less than $7 \%$.

Among the contemplated modifications lies an algorithm to automatically determine where and when the pilot should stop accelerating in order to take a curve at the maximum possible velocity. Also, this model could predict the behavior of many types of vehicles as it may be upgraded to 
work with AC motors and even fuel engines. With these modifications accurate prediction could be possible just by establishing the corresponding curves for torque and current (or fuel consumption rate) vs. angular velocity. For the latter case a variable mass should be also taken into account. These are some of the reasons why this program could become a very useful tool for analyzing many types of vehicles, focusing in racing applications.

\section{ACKNOWLEDGEMENTS}

We truly thank professors Jorge Aguirre and José Luis Gómez for encouraging us to develop the model and to publish it. We also thank each member of the Team for their constant collaboration.

\section{REFERENCES}

[1] Toyota-Electratón-LTH. (2013) History, retrieved 6 June 2013, from http://www.electraton.com. $\mathrm{mx} /$ historia.htm.

[2] SKF. (2013) Needle roller and cage assemblies, retrieved 6 June, 2013, from http://www.skf. $\mathrm{com} / \mathrm{ca} /$ en/products/bearings-units-housings/roller-bearings/needle-roller-bearings/needle-roller-and-cage-assemblies/index.html.

[3] White, F. (1998) Fluid Mechanics, 4th ed., McGraw Hill, New York, NY.

[4] Bettes, W. (1982) The Aerodynamic Drag of Road Vehicles-Past, Present, and Future, Engineering and Science 45, 4-10.

[5] Stratton, B. a. (2001) Etek Electric Motor System, retrieved 6 June, 2013, from http://www.robotmarketplace.com/products/images/etekmanual.pdf.

[6] Alltrax. (2008) AXE Performance products used for series wound or permanent magnet electric motors, retrieved 6 June, 2013, from http://www.alltraxinc.com/Products_AXE.html.

[7] Balsas, A. y. F. d. (2012) Full Power FP-75-650, retrieved 6 June, 2013, from http://www.afbalsas. com/index.php/component/content/article/19-productos/acumuladores/full-power/291-full-powerfp-75-650.

[8] Pascoe, P. a. A., A.. (2004) VRLA Battery Discharge Reserve Time Estimation, Power Electronics, IEEE Transactions 19, 1515-1522.

\section{ABOUT THE STUDENT AUTHORS}

Fernando León-Cázares is a senior student in the area of mechanical engineering in Instituto Tecnológico y de Estudios Superiores de Monterrey Campus Estado de México. He was the Electrathon Borregos CEM Racing's captain for two years and he has participated in a research group in the same institute dealing with the mathematical modeling of a plasma nitriding process. Daniel XoconostleLuna is a mechatronics engineering student who began to work full time in 2013.

\section{PRESS SUMMARY}

An Electrathon is a custom built electric vehicle, similar in appearance to a Go-Kart but powered by an electric motor. We developed a mathematical model to simulate the performance of such vehicle under different conditions so that it is possible to predict its velocity, acceleration and energy consumption at any point of any specific track. This allows for the optimization of different parameters of the vehicle to build the best Electrathon possible. 


\title{
Proteomic Study of Ribosomal Proteins from Escherichia coli, Saccharomyces cerevisiae, Bos taurus, Gallus gallus, and Oncorhynchus tshawytscha: Application in a Teaching Laboratory Setting
}

\author{
Yoshihiro Miura ${ }^{\dagger \neq}$, Eric Yeager ${ }^{\ddagger}$,James A. MacKenzie ${ }^{\ddagger}$, Kestutis Bendinskas ${ }^{\dagger}$ \\ ${ }^{\dagger}$ Department of Chemistry and ${ }^{\ddagger}$ Department of Biological Sciences, SUNY Oswego \\ Students:miura@oswego.edu,eay26@cornell.edu \\ Mentors: james.mackenzie@oswego.edu,kestutis.bendinskas@oswego.edu
}

\begin{abstract}
Ribosomes are central to protein synthesis and our understanding of ribosomes has advanced antibiotics research. The proteomic study of ribosomes presented here utilizes a combination of differential centrifugation and matrix assisted laser desorption/ionization-time of flight mass spectrometry (MALDI-TOF MS) to analyze ribosomes from various species in a teaching laboratory setting. Five biologically varied species were used: Escherichia coli (bacteria), Saccharomyces cerevisiae (yeast), Bos taurus (cow), Gallus gallus (chicken), and Oncorhynchus tshawytscha (Chinook salmon). Samples were lysed, ribosomes were isolated via ultracentrifugation using a discontinuous sucrose gradient and the individual protein subunits were separated via sodium dodecyl sulfate polyacrylamide gel electrophoresis. Tryptic digest and MALDI-TOF MS were then conducted on fifteen bands excised from the gel, and the mass spectra of both the whole protein sample and peptides were analyzed. Five out of these fifteen bands were positively identified as various ribosomal proteins, with two uncertain identifications. Additionally, three of the five positively identified proteins that travelled the same distance on the gel were determined to be orthologous. Finally, a class of 14 Biochemistry II students utilized these protocols, identified three ribosomal proteins and provided their evaluations of the ultracentrifugation-proteomics teaching laboratory.
\end{abstract}

\section{KEYWORDS}

Proteomics, MALDI-TOF MS, ultracentrifugation, ribosomes, teaching laboratory

\section{INTRODUCTION}

Ribosomes use the ribonucleic acid sequence of mRNA to synthesize proteins. ${ }^{1}$ Understanding ribosome structure is indispensable in endeavors such as the development of new antibiotics. ${ }^{2}$ Technological advances in gel-electrophoresis in combination with mass spectrometry allow scientists to understand the individual protein components of the ribosome. Nguyen-Lefebvre et al. successfully purified, identified, and quantified ribosomal proteins from human, rat, and chicken samples using a combination of techniques including two-dimensional-polyacrylamide gel electrophoresis (2D-PAGE), ultracentrifugation, matrix assisted laser desorption/ionization-time of flight mass spectrometry (MALDI-TOF MS), and liquid chromatography tandem mass spectroscopy (LC-MS/MS). ${ }^{3}$ Marquez et al., in a similar study, positively identified 128 out of 130 ribosomal proteins from the Archaean P. aerophilum and S. acidocaldarius, again using 2D-PAGE, ultracentrifugation, MALDI-TOF and LC-MS/MS. ${ }^{4} \mathrm{Xu}$ et al. performed a proteomic study of soybean leaves and identified a variety of metabolic and ribosomal proteins using the same techniques and instrumentation. ${ }^{5}$

The goal of this study was to successfully identify ribosomal proteins found in Escherichia coli, yeast, and in liver of cow, chicken, and salmon using a combination of ultracentrifugation and 
MALDI-TOF MS in a teaching laboratory setting. These five organisms were chosen for their availability and evolutionary variance. Past studies varied in the organisms and tissues from which ribosomes have been isolated. Some publications involved the extraction of bovine hepatic ribosomal proteins. ${ }^{6}$ Orvos et al. conducted a study to determine a protocol that would yield an optimal amount of salmon hepatic ribosomes. ${ }^{7}$ Nguyen-Lefebvre et al. successfully used this contemporary method to extract chicken ribosomes, but the study did not use liver. ${ }^{3}$ This study attempted to establish a unified procedure that would be compatible for extracting and analyzing cytosolic ribosomes from all five organisms in a teaching laboratory setting.

\section{PROCEDURE}

While it would have been convenient to homogenize each sample with the same procedure, the intrinsic differences between E. coli, yeast, and the liver samples required three different lysis protocols.

For E.coli, $1 \mathrm{~g}$ of sample was dissolved in $3 \mathrm{~mL}$ of solution A $(25 \mathrm{mM}$ Tris- $\mathrm{HCl}, 50 \mathrm{mM} \mathrm{KCl}$, $5 \mathrm{mM} \mathrm{MgSO}_{4}, 0.1 \mathrm{mM}$ EDTA, $7 \mathrm{mM}$ beta-mercaptoethanol, $250 \mathrm{mM}$ sucrose, $\mathrm{pH}$ 7.6) in a $15 \mathrm{~mL}$ centrifuge tube (reagents and supplies are from Thermo Scientific, Waltham, MA, unless otherwise specified). This solution was then sonicated using a Branson sonifier (Model 450, Branson Ultrasonics, VWR, Danbury, CT) for two minutes at setting 3 on a $30 \%$ cycle while on ice. This process causes localized low pressure regions that result in acoustic cavitation, ultimately lysing the cell.

For yeast, $1 \mathrm{~g}$ of sample was dissolved in $3 \mathrm{~mL}$ of solution $\mathrm{A}$, and homogenized with a French press (Sim-Aminco Spectronic Instruments, Rochester, NY) twice into a similar centrifuge tube. This device uses massive pressure to force the cells through a small opening, causing them to explode from the pressure gradient as they exit the press.

For the liver samples, a mortar and pestle were used to grind the samples with $4.5 \mathrm{~mL}$ of solution A and 0.5 grams of sea sand. The liver homogenate was then filtered through 8 layers of cheese cloth into a tube to remove crude cellular debris.

All five homogenates were then centrifuged for ten minutes at $4^{\circ} \mathrm{C}, 29,000 \mathrm{xg}$ in a centrifuge (RC6, Sorvall, Thermo Scientific) to pellet unlysed cells and cellular debris. The ribosome-containing supernatant was removed and centrifuged second time. The liver samples tended to accumulate a thin, undesirable layer of fat at the top of the tube which was removed with a pipette. Once the second round of centrifugation was complete, discontinuous sucrose gradients were prepared in 10 $\mathrm{mL}$ ultracentrifuge tubes: $2 \mathrm{~mL}$ of solution $\mathrm{C}$ (same composition as solution A but $2.0 \mathrm{M}$ sucrose) at the bottom of a tube, $2 \mathrm{~mL}$ of solution B (same as A but $1.5 \mathrm{M}$ sucrose), then $2.5 \mathrm{~mL}$ of supernatant from the homogenate on the top of a tube. The delineations of these layers can clearly be seen in Figure 1. The tubes were then placed in a T-890 fixed angle rotor, and spun in an ultracentrifuge (WX80, Sorvall, Thermo Scientific) at $180,000 \mathrm{xg}$ for 4 hours at $4^{\circ} \mathrm{C}$. 


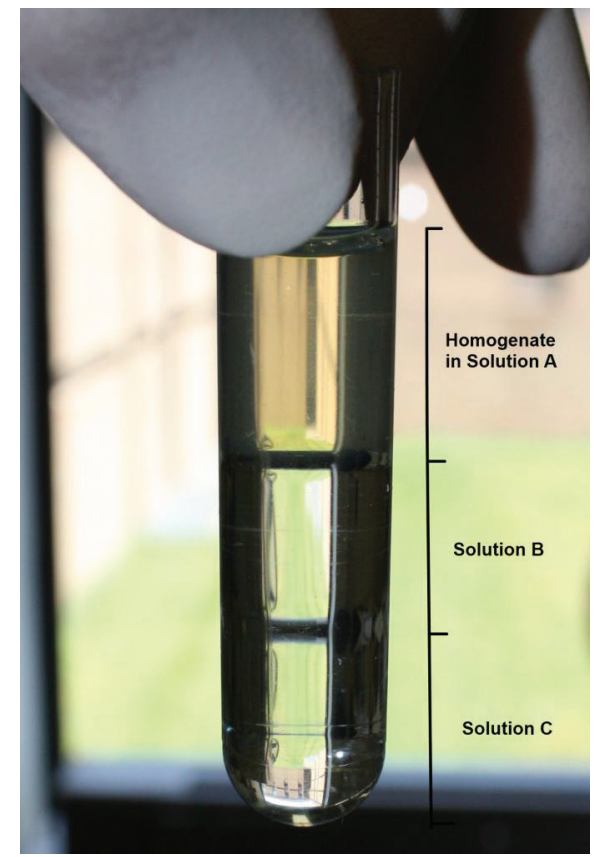

Figure 1. There is a clear delineation between the different layers of sucrose concentrations.

The supernatant was removed and pellets were resuspended in $100 \mu \mathrm{L}$ of water. UV-Vis spectrometry (Model 2000, NanoDrop, Thermo Scientific) was performed to confirm the presence of ribosomes in the samples. The expected A260/A280 absorbance ratio is approximately 1.8 to 1.9; protein concentration $(\mathrm{mg} / \mathrm{mL})=(1.55 \times \mathrm{A} 280)-(0.76 \times \mathrm{A} 260)$ (Equation 1$){ }^{8,9}$ This concentration is important to know so that the SDS-gel is not overloaded in the following step.

At this point in the study, the resuspended pellet contains at least 50 different proteins. Therefore, the protein mixture was separated using SDS-PAGE. Since we could not achieve good separation on a typical 2D gel due to the basic nature of ribosomal proteins, a premade $4-20 \%$ Tris- $\mathrm{HCl} 1 \mathrm{D}$ gradient gel (Criterion, BioRad, Hercules, CA) was used. The samples were first prepared in 2X SDS sample buffer (62.5 mM Tris-HCl, 69.4 mM SDS, $0.1 \mathrm{mM}$ bromophenol blue, $70 \mathrm{mM}$ beta-mercaptoethanol, $20 \% \mathrm{v} / \mathrm{v}$ glycerol) and then heated at $95^{\circ} \mathrm{C}$ for three minutes. Denatured proteins $(20 \mu \mathrm{g})$ from each sample were loaded along with several lanes of molecular weight markers. The gel was run at $200 \mathrm{~V}$ for one hour, washed twice for five minutes with water to remove excess SDS, and then stained with Bio-safe Coomassie (BioRad) to visualize the bands. Excess Coomassie dye was then removed from the background by washing the gel twice with water for five minutes.

Three bands from each sample lane were excised from the gel for further analysis. When selecting which bands to excise from the gel, we chose a band that seemed common among all of the species. Other bands were chosen based on how well they were separated from other bands so as to not mix proteins from other bands during excision. The excised gel pieces were placed in $1.6 \mathrm{~mL}$ protein-low-bind tubes (Eppendorf, Hamburg, Germany) with $200 \mu \mathrm{L}$ of destaining solution $\left(0.2 \%\right.$ ammonium bicarbonate in $50 \%$ acetonitrile) and incubated at $40^{\circ} \mathrm{C}$ for ten minutes to remove the Coomassie stain from protein bands. This step was repeated using fresh destaining solution until no dye remained visible. Stain removal is an essential step for successful digestion because Coomassie binds to lysine and arginine amino acid residues - the same residues at which the digestive enzyme trypsin cuts later in the procedure.

Forty $\mu \mathrm{L}$ of reducing buffer $(100 \mathrm{mM}$ dithiothreitol) was then added to the sample and incubated at $60^{\circ} \mathrm{C}$ for ten minutes to sever the disulfide bridges between cysteine residues. After cooling, 
the solution was removed and $40 \mu \mathrm{L}$ of alkylating buffer (100 mM iodoacetamide) was added and stored in the dark at room temperature for an hour. This step served to "cap" the now broken disulfide bridges with amide groups, preventing the bridges from reforming. Two hundred $\mu \mathrm{L}$ of additional destaining buffer was added and incubated at $37^{\circ} \mathrm{C}$ for 15 minutes to remove any remaining reducing or alkylating agents. The solution was removed, and $50 \mu \mathrm{L}$ of acetonitrile was added which dehydrated and shrank the gel pieces. The acetonitrile was removed, and the pieces were air-dried for ten minutes, and then $15 \mu \mathrm{L}$ of activated trypsin (Modified Porcine Gold, Promega, Madison, WI) was added. This caused the gel to rehydrate and swell, drawing the trypsin into the gel. After incubating for 15 minutes at room temperature, $25 \mu \mathrm{L}$ of digestion buffer $(0.4 \%$ ammonium bicarbonate) was added and the tubes were incubated at $37^{\circ} \mathrm{C}$ with gentle shaking overnight. This final step allowed the trypsin enzyme to cut the proteins at lysine and arginine to yield a mixture of peptides, which were now small enough to freely diffuse out of the gel.

Next, the tubes were placed in a water bath sonicator (FS20, Fisher Scientific, Pittsburg, PA) for 30 minutes to help peptides diffuse out of the gel and remove any peptides that may have bound to the tube walls. The peptide solutions, after being transferred to new tubes, were then vacuum-centrifuged to dryness at $60^{\circ} \mathrm{C}$ for an hour. The samples were then resuspended in $10 \mu \mathrm{L}$ of Solution 1 ( $0.1 \%$ trifluoroacetic acid) and water-bath sonicated again for 30 minutes to redissolve all the peptides. In the final preparatory step before MALDI-TOF, the peptides were concentrated and purified using C4 Zip-tips (Millipore, Billerica, MA). These specialized pipette tips contain a non-polar resin that binds peptides and facilitates the removal of salts and other impurities. The tips were prepared by aspirating $10 \mu \mathrm{L}$ of Solution $2(50 \%$ acetonitrile and $50 \% 0.1 \%$ trifluoroacetic acid) twice, then $10 \mu \mathrm{L}$ of Solution 1 twice. Subsequently, each sample, with their own Zip-tips, were aspirated and dispensed seven times. The peptides, along with some impurities, were now bound to the resin in the tip, leaving salts behind in solution. By aspirating Solution 1, any impurities that may have also bound to the resin were discarded while the peptides were still bound. Finally, the tip was aspirated and dispensed three times into a new tube containing only $4 \mu \mathrm{L}$ of Solution 2 , removing the peptides from the resin, and concentrating them in an extremely small volume. The isolated peptides were now ready for analysis by mass spectroscopy using MALDI-TOF (Autoflex II, Bruker Daltonics GmbH, Billerica, MA).

MALDI-TOF is a technique which measures the mass/charge ratio of a variety of different biomolecules. For whole, undigested proteins, samples must be prepared in a 1:4 ratio of sample to $1 \%$ sinapinic acid (Fluka Analytical, Sigma-Aldrich, St. Louis, MO) matrix solution, whereas digested peptides must be prepared in a 1:4 ratio of sample to $1 \%$ a-hydroxycinnamic acid (Fluka Analytical) matrix solution. Both the total protein sample (unfractionated by gel electrophoresis) and the individually digested bands from the SDS-PAGE were analyzed on the MALDI-TOF. Two $\mu \mathrm{L}$ of each sample/matrix mixture were spotted onto the MALDI target plate along with protein and peptide standards to properly calibrate the instrument, then air dried. The MALDI-TOF utilizes a laser fired at the samples under a vacuum causing the matrix to absorb much of the laser's energy ionizing the sample. The matrix also gives all of these proteins/peptides predominantly +1 charge, and they travel through the vacuum due to a high voltage within the instrument, size differences being resolved by the time of flight. It is typical when using MALDI-TOF to vary the power of the laser and the number and location of shots - these parameters must be adjusted by the user during each analysis. 


\section{RESULTS}

Purified ribosomes were analyzed via UV-Vis spectroscopy of the dissolved pellets from the ultracentrifugation step (Table 1). Liver samples required a 100-fold dilution and the E.coli and yeast a 20 -fold dilution before analysis. All samples had an A260/A280 of approximately 2.0 and yielded a protein content between $9-10 \mu \mathrm{g} / \mathrm{mL}$, except for the salmon liver, which was $2 \mu \mathrm{g} / \mathrm{mL}$.

\begin{tabular}{|c|c|c|c|c|}
\hline Sample & Dilution & A260/A280 & A280 & $\begin{array}{c}\text { Protein } \\
\text { Concentration } \\
(\boldsymbol{\mu g} / \mathbf{m L})\end{array}$ \\
\hline Beef & 100 & 1.96 & 0.15 & 9 \\
\hline Chicken & 100 & 1.99 & 0.26 & 10 \\
\hline Salmon & 100 & 2.02 & 0.16 & 2 \\
\hline E.coli & 20 & 1.96 & 0.15 & 9 \\
\hline Yeast & 20 & 1.99 & 0.26 & 10 \\
\hline
\end{tabular}

Table 1. Absorbance Data Retrieved from UV-Vis Spectroscopy.

A representative gel of proteins from the pellets is shown in Figure 2, with the bands that were excised for protein identification being denoted by the boxes.

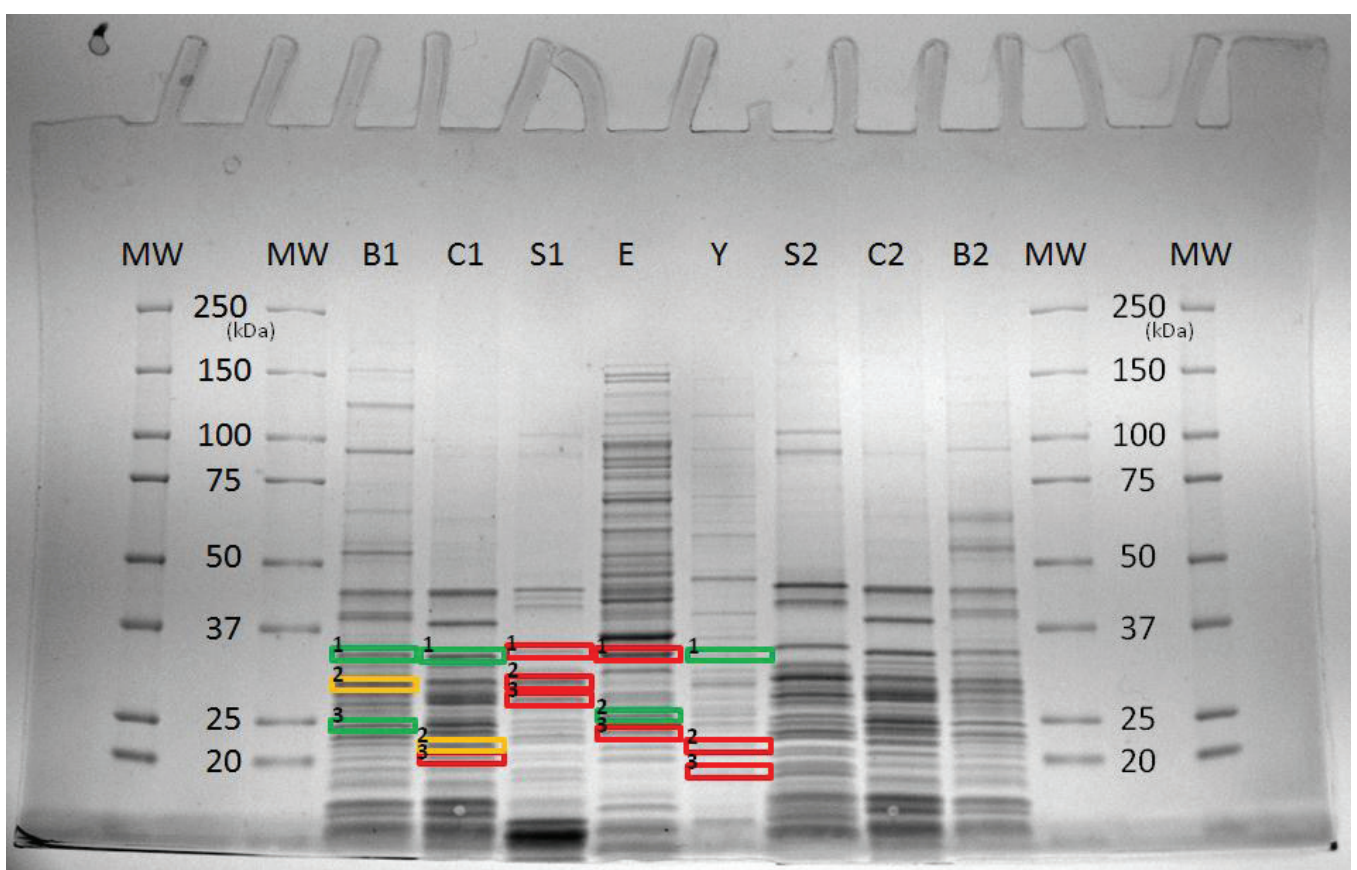

Figure 2. Molecular weight markers (MW) were separated via 4-20\% SDS-PAGE along with ribosomal proteins isolated from beef $(\mathrm{B} 1, \mathrm{~B} 2)$, chicken $(\mathrm{C} 1, \mathrm{C} 2)$, salmon $(\mathrm{S} 1, \mathrm{~S} 2)$, E. coli $(\mathrm{E})$, and yeast $(\mathrm{Y})$. The rectangles indicate which bands were excised and analyzed via tryptic digest and MALDI-TOF. The green rectangles were successfully identified with $95 \%$ confidence, the orange rectangles have questionable results, and the red rectangles were not successfully identified. The smaller numbers next to the rectangles indicate the band's assigned number for easier reference. 
Figure 3 depicts the total protein spectra collected for each sample individually as well as a combined overlay. For the digested proteins in individual bands from the gel, the resulting peptide spectra were submitted to an online database (Swiss-Prot) for identification.
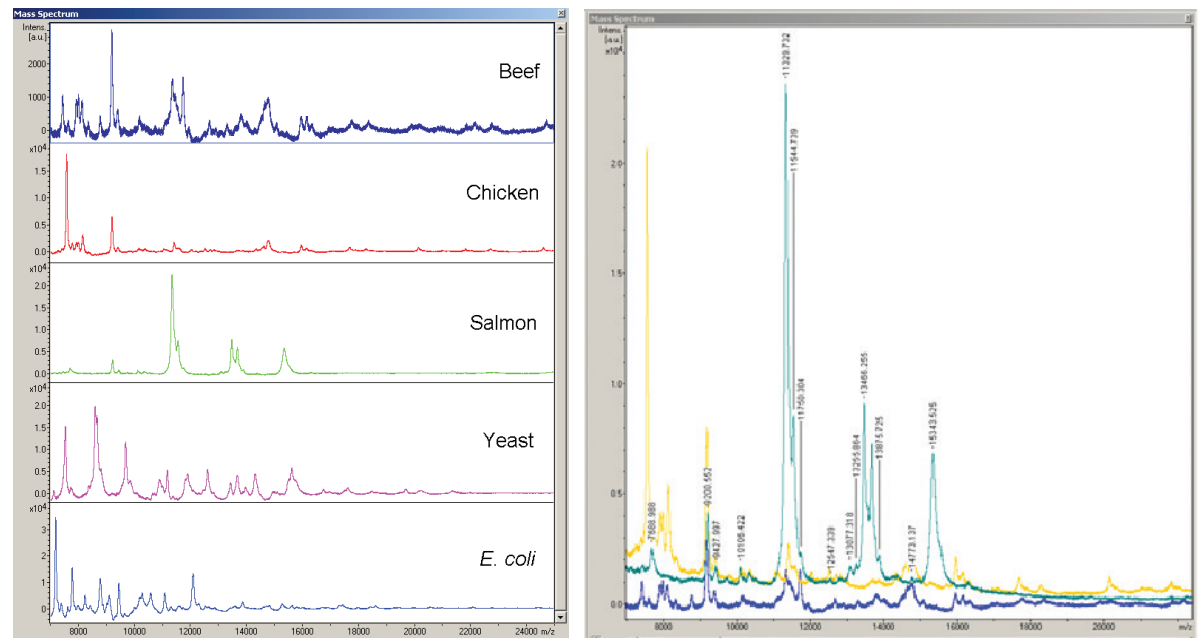

Figure 3. The total protein mass spectra for each organism, displayed side by side (left panel). The total protein displayed in an overlay manner: beef $=$ dark blue, chicken $=$ yellow, salmon $=$ aquamarine (right panel). $\mathrm{X}$ axis is the measured mass to charge ratio for proteins and y axis is intensity, which represents abundance of proteins. 
Figure 4 shows an example digest spectrum from a single band. Certain parameters of the database search were adjusted such as the taxonomy (the biological classification of the organism from which the sample was prepared), the peptide tolerance (a measure of the accuracy of the peak measurements) and the number of missing trypsin cleavages (the value of zero assumes that all cuts were perfectly complete, while 1 assumes that an occasionally cut was missed). The statistical significance of the search results were determined by the MOlecular Weight SEarch (MOWSE) score - the higher the value, the more significant the result. MOWSE score depends on the selected significance, the closeness of the hit, the size of the data base, etc. Figure 4 also shows the alignment of the spectra with the sequence of the protein. In that particular example, $68 \%$ intensity coverage and $45.1 \%$ sequence coverage were achieved and eighteen peaks were matched. The MOWSE score of 84 was achieved while the $95 \%$ significance for that set of search parameters was calculated to be at the MOWSE score of 70. A positive hit in a database provides detailed information about each protein, such as its isoelectric focusing point, molecular weight, amino acid sequence, number of possible peaks and the percent sequence coverage by this particular digest.

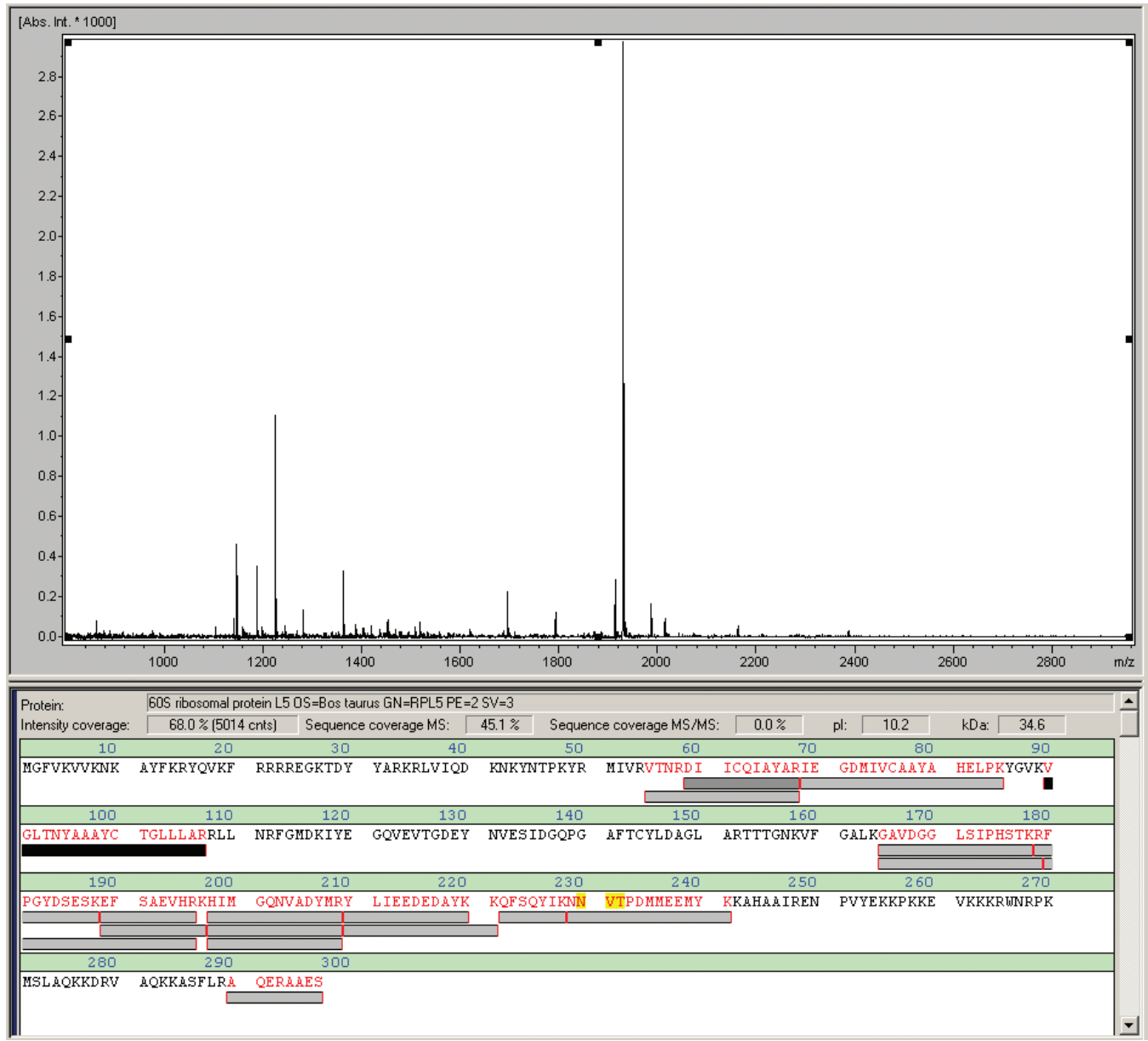

Figure 4. A representative image of the MALDI-TOF digest spectra (top section) and sequence alignment (bottom section) for beef band 1 . Eighteen peaks matched, and $68 \%$ intensity coverage and $45.1 \%$ sequence coverage were achieved. The protein was identified to be 60S Ribosomal Protein L5 (Bos taurus) and achieved the highest MOWSE score among all identified samples with a value of 84 . $\mathrm{X}$ axis is the measured mass to charge ratio for peptides and $\mathrm{y}$ axis is intensity, which represents the abundance of peptides. 
Table 2 summarizes the parameters used and the significant results collected from the database searches. A total of five different ribosomal proteins were identified, including three from beef liver (60S proteins L5 and L15, 40S protein S3), two from chicken liver (60S protein L5, 40S protein S9), one from E. coli (30S protein S4), and one from yeast (60S protein L5). We were not able to significantly identify any of the three bands from Figure 2 for salmon liver.

\begin{tabular}{|c|c|c|c|c|c|c|c|}
\hline $\begin{array}{c}\text { Band } \\
\text { Excised } \\
\text { from Fig } 2\end{array}$ & $\begin{array}{l}\text { Taxonomy } \\
\text { used in } \\
\text { search }\end{array}$ & $\begin{array}{l}\text { Peptide } \\
\text { Tolerance }\end{array}$ & Cleavages & Protein & $\begin{array}{l}\text { MOWSE } \\
\text { Score }\end{array}$ & $\begin{array}{c}\text { Mass of } \\
\text { Protein } \\
(\mathrm{kDa})\end{array}$ & $\begin{array}{c}\text { Approximate } \\
\text { MW Deduced } \\
\text { by PAGE } \\
\text { Markers (kDa) }\end{array}$ \\
\hline \multirow[t]{2}{*}{ Beef 1} & \multirow[t]{2}{*}{ All Entries } & \multirow[t]{2}{*}{0.8308} & \multirow[t]{2}{*}{1} & $\begin{array}{l}60 \text { S Ribosomal } \\
\text { Protein L5 }\end{array}$ & \multirow[t]{2}{*}{84} & \multirow[t]{2}{*}{34.551} & \multirow[t]{2}{*}{$34-35$} \\
\hline & & & & (Bos taurus) & & & \\
\hline \multirow[t]{2}{*}{ Beef 2} & \multirow[t]{2}{*}{$\begin{array}{c}\text { Other } \\
\text { Mammalian }\end{array}$} & \multirow[t]{2}{*}{0.4742} & \multirow[t]{2}{*}{1} & $\begin{array}{c}40 \text { S Ribosomal } \\
\text { Protein S3 }\end{array}$ & \multirow[t]{2}{*}{69} & \multirow[t]{2}{*}{26.984} & \multirow[t]{2}{*}{$30-32^{*}$} \\
\hline & & & & (Bos taurus) & & & \\
\hline \multirow[t]{2}{*}{ Beef 3} & \multirow[t]{2}{*}{$\begin{array}{c}\text { Other } \\
\text { Mammalian }\end{array}$} & \multirow[t]{2}{*}{0.3745} & \multirow[t]{2}{*}{1} & \begin{tabular}{|c|} 
60S Ribosomal \\
Protein L15 \\
\end{tabular} & \multirow[t]{2}{*}{57} & \multirow[t]{2}{*}{24.245} & \multirow[t]{2}{*}{$24-25$} \\
\hline & & & & (Bos taurus) & & & \\
\hline \multirow[t]{2}{*}{ Chicken 1} & \multirow[t]{2}{*}{ All Entries } & \multirow[t]{2}{*}{0.87} & \multirow[t]{2}{*}{2} & $\begin{array}{l}60 \text { S Ribosomal } \\
\text { Protein L5 }\end{array}$ & \multirow[t]{2}{*}{75} & \multirow[t]{2}{*}{34.298} & \multirow[t]{2}{*}{$34-35$} \\
\hline & & & & (Gallus gallus) & & & \\
\hline \multirow[t]{2}{*}{ Chicken 2} & \multirow[t]{2}{*}{$\begin{array}{c}\text { Bony } \\
\text { Vertebrates }\end{array}$} & \multirow[t]{2}{*}{0.4774} & \multirow[t]{2}{*}{0} & \begin{tabular}{|c|}
40 S Ribosomal \\
Protein S9 \\
\end{tabular} & \multirow[t]{2}{*}{$45 \dagger$} & \multirow[t]{2}{*}{22.635} & \multirow[t]{2}{*}{$22-23$} \\
\hline & & & & (Gallus gallus) & & & \\
\hline \multirow[t]{2}{*}{ E. coli 2} & \multirow[t]{2}{*}{ E. coli } & \multirow[t]{2}{*}{0.9937} & 1 & \begin{tabular}{|c|}
30 S Ribosomal \\
Protein S4 \\
\end{tabular} & 62 & 23.512 & $25-26$ \\
\hline & & & & (E.coli) & & & \\
\hline Yeast 1 & Yeast & 0.7023 & 2 & \begin{tabular}{|c|}
60 S Ribosomal \\
Protein L5 \\
\end{tabular} & 63 & 33.751 & $34-35$ \\
\hline & & & & (S. cerevisiae) & & & \\
\hline
\end{tabular}

Table 2. Significant Hits Collected from SwissProt Database. 
For each step outlined previously, a multitude of conditions were screened to find the optimum search parameters. All experiments were completed in triplicate. After the protocols were worked out, Biochemistry Laboratory II students ran the experiment in the Spring semester of 2012. These 14 students successfully identified 3 ribosomal proteins and provided feedback summarized in Table 3.

\begin{tabular}{|l|c|c|}
\hline Evaluation Questions & Average & SD \\
\hline $\begin{array}{l}\text { As a consequence of performing this laboratory experiment, how well do you under- } \\
\text { stand the difference between the "reductionist approach" to biology as opposed to } \\
\text { "systems" biology (e.g. protein vs proteomics)? }\end{array}$ & 3.8 & 0.7 \\
\hline How well do you understand the principles of proteomics? & 4.1 & 0.6 \\
\hline How well do you understand the concept of ultracentrifugation? & 4.7 & 0.5 \\
\hline $\begin{array}{l}\text { How well do you understand the processes of protein separation and modification } \\
\text { (prior to their analysis)? }\end{array}$ & 4.3 & 0.6 \\
\hline How well do you understand the principles of MALDI-TOF spectroscopy? & 4.3 & 0.9 \\
\hline How well do you understand the concept of protein mass fingerprinting? & 3.8 & 0.7 \\
\hline $\begin{array}{l}\text { How well do you understand the parameters involved in performing protein database } \\
\text { searches? }\end{array}$ & 3.5 & 1.1 \\
\hline Were the laboratory directions presented in a clear manner? & 4.4 & 0.6 \\
\hline Did you find this laboratory more interesting than a typical laboratory experiment? & 4.1 & 0.9 \\
\hline Would you recommend continuing this laboratory as part of this course in the future? & 4.5 & 0.9 \\
\hline Possible Answers & & \\
\hline (Not clear) 1 2 3 4 (Very clear) & & \\
\hline (Less interesting) 1 2 3 5 (More interesting) & & \\
\hline (Not inclined to recommend) 1 2 3 5 (Strongly inclined to recommend) & & \\
\hline Student-Identified Proteins & & \\
\hline Yeast 60S Ribosomal Protein (2X) & & \\
\hline Yeast 40S Ribosomal Protein & & \\
\hline E.coli 30S Ribosomal Protein & & \\
\hline
\end{tabular}

Table 3. Student results and evaluations of the ultracentrifugation-proteomics laboratory.

\section{DISCUSSION}

Our data in Table 1 indicates that we successfully purified ribosomes. The typical A260/A280 ratio, which was about 2.0, suggests the presence of ribonuclear proteins in the precipitated pellet. The downstream analysis via SDS-PAGE (Figure 2) and MALDI (Table 2, Figure 4) confirm the presence of ribosomal proteins. The yield of salmon ribosomal proteins was lower than in other species. When comparing the total protein spectra (Figure 3), it can be seen that even though the spectra are not identical, there are some similarities between them, particularly among the eukaryotes. Comparison between samples should be based on the presence or absence of peaks at particular mass/charge ratios, and not their intensities. Intensity is defined by the amount of protein present and the number of shots collected.

The similarities are strongest between the beef and chicken, but fish share some peaks with them as well. This is likely due to the evolutionary relationships between the three organisms-while they may be different species, they are all animals that belong to the phylum Chordata and on a cellular level they have many comparable characteristics. The fact that all living organisms require ribosomes to produce proteins would make these structures well-conserved among organisms, 
especially those which are evolutionarily close to each other. The peak similarity was not observed when comparing these three eukaryotes to another eukaryote, yeast. These differences are not surprising because yeast is a much simpler eukaryote-a single-celled organism of the kingdom Fungi. The similarities are also absent when comparing yeast to single-celled bacteria E. coli. This difference is likely attributed to the evolutionary divide between eukaryotes and prokaryotes which is highlighted by the different sizes of the overall ribosome for each group. ${ }^{1}$

After searching through the Swiss-Prot database, six statistically significant hits with a 95\% confidence interval were found (Figure 4, Table 2). Unfortunately, none of the bands excised from salmon produced significant hits, but conversely all three bands from beef yielded positive results. Upon closer inspection of the data, the result for Band 2 in beef may not have been a logical find. The database identified Band 2 as the 40S Ribosomal Protein S3, with a mass of approximately $27 \mathrm{kDa}$. When the location of the band is inspected on the SDS gel using the molecular weight markers as a reference, the band falls at about $32-34 \mathrm{kDa}$, which could be due to post-translational modifications. In this case, it is difficult to determine whether this band is truly the $40 \mathrm{~S}$ Ribosomal Protein S3. An additional LC-MS/MS study can be performed to determine whether this match is a false positive or not.

Out of the three chicken bands, only Band 1 was positively identified as a ribosomal protein. However, it is worth mentioning that Band 2 was rather close to being a significant hit. MOWSE scores can sometimes be improved by imposing stricter search parameters in peptide tolerance, but these efforts were insufficient to obtain a positive match with $95 \%$ confidence. The mass of $40 \mathrm{~S}$ Ribosomal Protein S9 does fall within the expected protein mass range from the gel, so there is a possibility that this band is in fact this protein. However, without more information to submit to the database, this cannot be confirmed.

One final compelling point of interest was that the significant results seen in Band 1 for beef, chicken and yeast were all identified as 60S Ribosomal Protein L5. The fact that these individually processed protein bands that travelled the same distance on the gel yielded the same exact ribosomal protein from different species is a powerful indicator that speaks to the validity of this experiment. Thus, we concluded that it was possible to successfully extract and identify ribosomal proteins from various species using ultracentrifugation and MALDI-TOF.

Finally, we replicated these methods in a Biochemistry II teaching laboratory. To the best of our knowledge the current literature lacks the description of a teaching laboratory that applies ultracentrifugation. This enables institutions with such instrumentation to try this experiment. The student and instructor's manuals are available from the corresponding author. As can be seen by student evaluations (Table 3), the experiment was well liked by students with the average valuation for all questions asked being 4.1 out of 5 . Future work in this study will include identification of all bands by LC-MS/MS with the help of our collaborators, repeating analysis of the bands via MALDI-TOF and the improvement of student protocols to ensure better identification rate and reproducibility.

\section{ACKNOWLEDGEMENTS}

We thank SUNY Oswego, specifically the Scholarly and Creative Activity Committee as well as the Provost's office for their support. We thank NSF-MRI for funds for the acquisition of an ultracentrifuge for undergraduate research training and teaching.

\section{SUPPLEMENTAL INFORMATION}

Supplemental material may be found online in PDF format alongside the associated article at www.ajuronline.org. 


\section{REFERENCES}

[1] Alberts, B., Wilson, J. H., and Hunt, T. (2008) Molecular biology of the cell, 5th ed., Garland Science, New York.

[2] Bulkley, D., Johnson, F., and Steitz, T. A. (2012) The antibiotic thermorubin inhibits protein synthesis by binding to inter-subunit bridge $\mathrm{B} 2 \mathrm{a}$ of the ribosome, $\mathrm{J} \mathrm{Mol} \mathrm{BiO/} \mathrm{416,571-578.}$

[3] Nguyen-Lefebvre, A. T., Gonin-Giraud, S., Scherl, A., Arboit, P., Granger, L., Sanchez, J. C., Diaz, J. J., Gandrillon, O., and Madjar, J. J. (2011) Identification of human, rat and chicken ribosomal proteins by a combination of two-dimensional polyacrylamide gel electrophoresis and mass spectrometry, Journal of proteomics $74,167-185$.

[4] Marquez, V., Frohlich, T., Armache, J. P., Sohmen, D., Donhofer, A., Mikolajka, A., Berninghausen, O., Thomm, M., Beckmann, R., Arnold, G. J., and Wilson, D. N. (2011) Proteomic characterization of archaeal ribosomes reveals the presence of novel archaeal-specific ribosomal proteins, J Mol Biol 405, $1215-1232$

[5] Xu, C., Garrett, W. M., Sullivan, J., Caperna, T. J., and Natarajan, S. (2006) Separation and identification of soybean leaf proteins by two-dimensional gel electrophoresis and mass spectrometry, Phytochemistry 67, 2431-2440.

[6] Matthews, D. E., Hessler, R. A., Denslow, N. D., Edwards, J. S., and O’Brien, T. W. (1982) Protein composition of the bovine mitochondrial ribosome, J Bio/ Chem 257, 8788-8794.

[7] Orvos, D. R., Sellers, C. M., Jr., and Garrison, N. E. (1987) Optimal isolation conditions for rainbow trout (Salmo gairdneri) hepatic ribosomes, Comparative biochemistry and physiology. $B$, Comparative biochemistry 88, 141-145.

[8] Warburg, O., Christian, W. (1942) Isolierung und kristallisation des garangsferementens enolase, Biochemical Z 374-421, 374-421.

[9] Layne, E. (1957) Spectrophotometric and turbidimetric methods for measuring proteins, Methods Enzymol. 3, 447-454.

\section{ABOUT THE STUDENT AUTHORS}

Yoshihiro Miura graduated from SUNY Oswego in December 2012 with a BS in Biochemistry and Biology. He chose to get his D.P.T. degree at Columbia University, NYC, New York.

Eric Yeager graduated from the State University of New York (SUNY) at Oswego in May, 2010 with a BS degree in Zoology (minor in Chemistry). He is currently living in Ithaca, NY, while attending the New York State College of Veterinary Medicine at Cornell University pursuing his Doctorate in Veterinary Medicine (DVM).

\section{PRESS SUMMARY}

Ribosomes are central to protein synthesis and our understanding of ribosomes has advanced antibiotics research. We purified ribosomes and then ribosomal proteins and identified some of those proteins in five species: bacteria, yeast, salmon, chicken, and cow. We taught these advanced techniques to a class of biochemistry students. We suggest that other biochemists could use our protocols for teaching ultracentrifugation and proteomics in their teaching laboratories. 


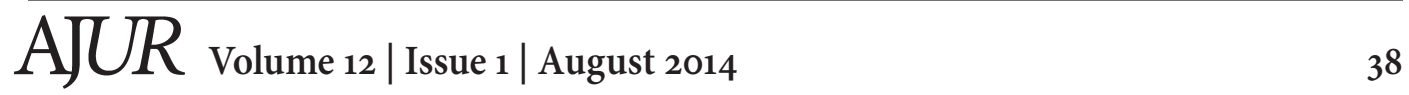




\title{
A Cellular Automaton Model for Traffic Flow - Investigating the Effect of Turning
}

\author{
Tracy Finner ${ }^{\dagger}$ and Matthew A. Beauregard ${ }^{\ddagger}$ \\ ${ }^{\dagger}$ Department of Mathematics, University of Arizona, $A Z$ \\ ${ }^{\ddagger}$ Department of Mathematics, Baylor University, Waco, TX \\ *Mentor: beauregama@sfasu.edu
}

\begin{abstract}
A cellular automaton model is proposed, modeling vehicular traffic flow on a two dimensional lattice in which the vehicles turn at an intersection with a given probability. It is shown that the introduction of turning reduces the long-term average velocity, and can be predicted by a power law depending on the probability of a vehicle turning and the density of cars. The reduction in speed decreases rapidly once the light cycle length surpasses a certain threshold, the value of which can be predicted from the observed power law.
\end{abstract}

\section{KEYWORDS}

cellular automaton, traffic flow, traffic light strategy, turning, dynamical systems, power law

\section{INTRODUCTION}

It is an everyday occurrence in North America to be influenced by traffic. In many cities congestion of traffic is attenuated by the introduction of highways or multiple lanes increasing the overall capacity of vehicles a network of roads can carry. However this is not a tractable solution for many cities that do not have affordable space to accommodate such an improvement. Hence there is a need to optimize traffic flow given the current network of roads through speed limits, roundabouts, one-way traffic, and most importantly, choosing the appropriate lighting strategy. Still, these alternatives require a large capital investment which should be done without adequate study of the effect these changes will have on the overall vehicular flow. Experimentation is not only costly but may lead to undesirable results. A more cost-effective approach is to study each effect through realistic mathematical modeling that incorporates probabilistic features of human behavior.

Traffic flow can either be studied macroscopically, mesoscopically, or microscopically (see Kerner ${ }^{10}$ for a complete exposition of approaches). Macroscopic models have been studied by many authors. $1,2,4,9,11,13$ A typical approach in the macroscopic model is to view the vehicles as a continuum and model them similar to that of a one-dimensional compressible fluid. The conservation of mass and momentum take the form of first order partial differential equations with the density and velocity of the vehicles serving as unknowns. In the mesoscopic or kinetic models, originally introduced by Prigogine, ${ }^{14}$ the governing equations are based on the Boltzman type kinetic equations. In these models a relaxation term is introduced in combination with a slowing down term to describe interactions due to braking of vehicles.

The cellular automaton (CA) models have been designed to study traffic flow at the microscopic level and are the basis of the model used in this paper. Each vehicle is viewed as a particle far from equilibrium and updated in parallel according to certain rules. The interactions of the vehicles can be complex, thus the particular effect of cars turning onto cross streets is examined in this paper. The authors recommend Chowdhury ${ }^{6}$ and Gartner and Stamatiadis ${ }^{8}$ for an overview of current CA models under study, and Chowdhury ${ }^{7}$ for a more thorough review. In this paper we introduce an extension to the Biham-Middleton-Levine $(\mathrm{BML})^{3}$ model and investigate the effect a turning 
vehicle has on the optimal light cycle length for a synchronous lighting strategy at a particular density. The reduction in the average velocity can be predicted using a newly developed power law.

The paper is organized as follows. The traffic model is introduced in the next section. Integrating turning necessitates a greater interaction among the vehicles as in previous models. Results of the model are given in section 3. It is observed that the flow is reduced as a consequence of turning vehicles and reduction in the average velocity can be predicted using a power law that depends on the density of cars and the turning velocity. Concluding remarks are given in section 4 .

\section{CELLULAR AUTOMATON MODEL}

The model presented uses a two-dimensional square lattice where each cell can either be empty or occupied by a vehicle traveling east or north. The authors consider single, one-way traffic lanes without allowing the overtaking of vehicles. Two-way traffic flow is not considered. There exists a traffic signal at each intersection of north and eastbound lanes and the behavior (green or red) is controlled by a global synchronized light strategy. In this strategy the traffic light is uniform in each direction, for example, green lights for all eastbound traffic simultaneously, then switched to a red light for all eastbound traffic, and green to northbound streets. This greatly simplifies the ability to investigate the dynamics as periodic boundary conditions reduce the lattice of roads to the case of one eastbound and northbound intersection. The orientation of the light is switched at a user-specified time step, $T$, herein referred as the light cycle length.

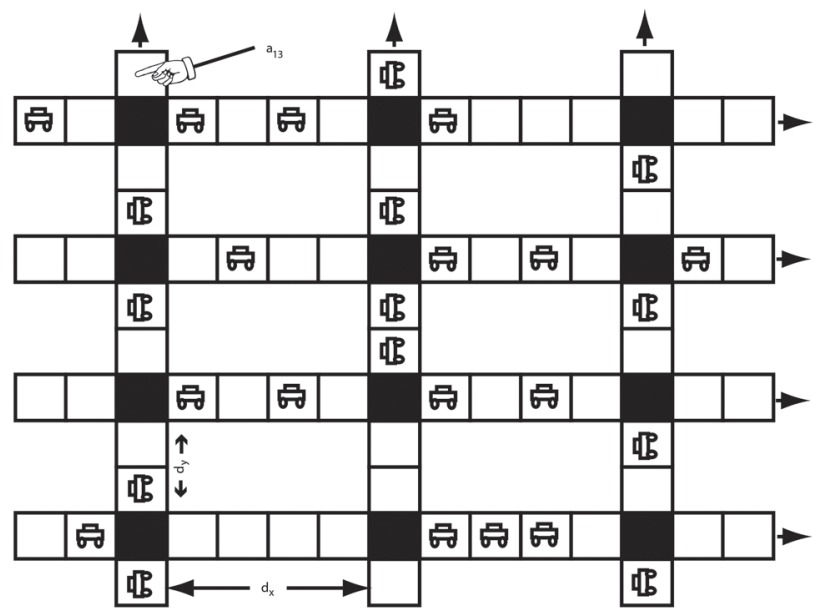

Figure 1. The vehicles are initially assigned random positions and velocities. The vehicles are evenly distributed among the roads. At each intersection a light exists that is either green or red for the oncoming eastbound or northbound traffic. The positions of the vehicles are associated with a particular cell's location $a_{i j}$. Parameters used: $N_{x}=4, N_{y}=3, N_{v}=28, d_{x}=4, d_{y}=2$.

There are $N_{x}$ and $N_{y}$ eastbound and northbound lanes, respectively. The total number of cars, $N_{v}$, are evenly distributed among the eastbound and northbound lanes. Periodic boundary conditions at the edge of the lattice are considered, hence the value of $N_{v}$ is conserved. The density of cars on a given lattice is

$$
\rho=N_{v} /\left(N_{x} N_{y}\left(d_{x}+d_{y}+1\right)\right)
$$

where $d_{x}$ and $d_{y}$ are the number of cells between northbound and eastbound lanes, respectively, and the denominator is the total capacity of the network. Each cell's location is stored in a sparse position matrix $a_{i j}$. For illustrative purposes the lattice for $N_{x}=4, N_{y}=3, N_{v}=28, d_{x}=4$, and $d_{y}=2$ is shown in Fig. 1. 
The value $d_{n}$ is the distance between vehicles $x_{n}$ and $x_{n+1}$ on the same road, while the value $\widetilde{d}_{n}$ is the distance to the next light plus the distance to the next vehicle on the next road perpendicular to the current one. If $d_{n}$ is one then the next vehicle is in the next adjacent cell. The distance from the current vehicle to the next intersection is given by $s_{n}$. An illustrative cartoon is shown in Fig. 2, in which $d_{n}=3, \widetilde{d}_{n}=4$, and $s_{n}=2$.

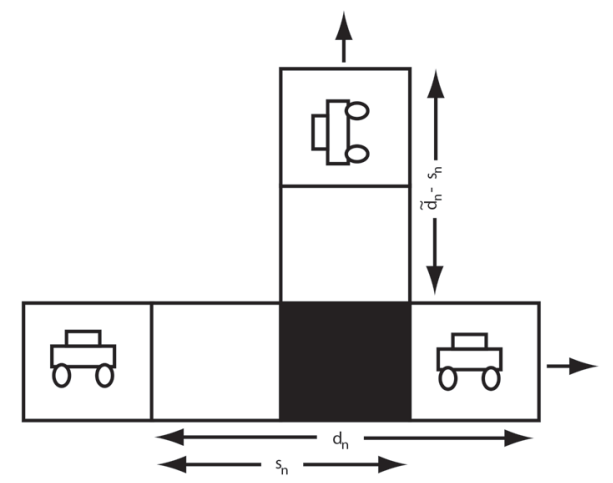

Figure 2. A schematic of vehicles near an intersection. The coordinates of each car are stored in a sparse matrix $a_{i j}$ which makes the calculations easier to compute.

The indices $i$ and $j$ refer to the cell location which is mapped to the vehicle $x_{n}$.

Each vehicle's velocity takes on discrete values of $0,1,2, \ldots, v_{\max }$. The vehicles are initially assigned random positions and velocities. No vehicle is initially placed in an intersection and no more than one vehicle may occupy a cell. The vehicle's positions and velocities are updated in parallel by applying the following rules at each time step:

Step 1: Acceleration

$$
v n \rightarrow \min \left(v_{n}+1, v_{\max }\right) .
$$

Step 2: Traffic Light, Turning, Collision Prevention

Case 1: IF INTERSECTION FLAG ON, i.e. CAR IN INTERSECTION (Turn FLAG OFF)

(a) If traffic light is red in front of $n^{\text {th }}$ vehicle then

$$
v_{n} \rightarrow \min \left(v_{n}, v_{\text {turn }}, d_{n}-1, s_{n}-1\right)
$$

(b) Otherwise traffic light is green in front of $n^{\text {th }}$ vehicle

i. If the next two cells directly past next intersection are occupied then

$$
v_{n} \rightarrow \min \left(v_{n}, v_{\text {turn }}, d_{n}-1, s_{n}-1\right)
$$

ii. Otherwise

$$
v_{n} \rightarrow \min \left(v_{n}, v_{\text {turn }}, d_{n}-1\right)
$$




\section{Case 2: IF INTERSECTION FLAG OFF}

(a) Random turning with probability $p_{\text {turn }}$

i. If traffic light is red in front of $n^{\text {th }}$ vehicle then

$$
v_{n} \rightarrow \min \left(v_{n}, d_{n}-1, s_{n}-1\right) .
$$

ii. Otherwise traffic light is green in front of $n^{\text {th }}$ vehicle

A. If $s_{n} \geq v_{\text {turn }}$ then $v_{n} \rightarrow \min \left(v_{n}, d_{n}-1, s_{n}\right)$

- If updated velocity $v_{n}=s_{n}$ then INTERSECTION FLAG ON

B. Otherwise $s_{n}<v_{\text {turn }}$ then $v_{n} \rightarrow \min \left(v_{n}, v_{\text {turn }}, d_{n}-1, \widetilde{d}_{n}-1\right)$.

- If updated velocity $v_{n}>s_{n}$ then TURNING FLAG ON

(b) Car not selected for turning

i. If traffic light is red in front of $n^{\text {th }}$ vehicle then

$$
v_{n} \rightarrow \min \left(v_{n}, d_{n}-1, s_{n}-1\right) .
$$

ii. Otherwise traffic light is green in front of $n^{\text {th }}$ vehicle

A. If the next two cells directly past next intersection are occupied then

$$
v_{n} \rightarrow \min \left(v_{n}, d_{n}-1, s_{n}-1\right) .
$$

B. Otherwise

$$
v_{n} \rightarrow \min \left(v_{n}, d_{n}-1\right) .
$$

Step 3: Random slowdown with probability $p_{\text {slow }}$

$$
v_{n}=\max \left(v_{n}-1,0\right) .
$$

Step 4: Update Position

Case 1: IF TURNING FLAG ON (Turn FLAG OFF)

(a) If Eastbound turning to Northbound lane then

$$
\mu=i+s_{n}, v=j-\left(v_{n}-s_{n}\right)
$$

(b) Otherwise Northbound turning to Eastbound lane

$$
\mu=i+\left(v_{n}-s_{n}\right), v=j+s_{n}
$$

Case 2: IF TURNING FLAG OFF

(a) If Eastbound vehicle then

$$
\mu=i, v=j+v_{n}
$$

(b) Otherwise Northbound vehicle then

$$
\mu=i-v_{n}, v=i
$$

Update Position: $a_{i j} \rightarrow a_{\mu v}$. 
In step 1 each vehicle is allowed to accelerate provided it is not at the maximum velocity. This refers to the natural human tendency to want to drive as fast as allowed. Speed is incremented by one at each time step that the car is allowed to accelerate.

Step 2 contains the primary interaction among the vehicles. This step is very similar to that found in the algorithm in Brockfeld et al., ${ }^{5}$ differing in that each vehicle can be selected for turning with a probability of $p_{\text {turn }}$. Turning only begins if the next traffic light is green. In this situation, the vehicle's speed is taken to be the minimum of its current velocity, the user-prescribed turning velocity $v_{\text {turn }}$, the distance to the next vehicle on the current road, or the distance to the next vehicle on the road it will be turning on to. Once a car, $x_{n}$, at position $a_{i j}$ is selected to turn, there are three distinct situations which can occur.

First, in the event the car is currently in an intersection, it will commence turning upon arriving at the next traffic light on the current road it is on. This restriction is based on the reality that a car in the middle of an intersection can not make a decision to turn without updating its velocity prior to arriving at the intersection. The Intersection Flag is used to denote cars that are in the intersection at which they are scheduled to turn. Secondly, consider $s_{n} \geq v_{\text {turn }}$. If the traffic signal is red then the vehicle proceeds as in the turn-free model, $v_{n} \rightarrow \min \left(v_{n}, d_{n}-1, s_{n}-1\right)$. When the traffic signal is green the vehicle will not be able to complete the turn at the current time-step and, at best, will become situated in the intersection itself with speed $s_{n}$. At the next time step the car will complete its turn with a velocity of $\min \left(v_{n}, v_{\text {turn }}, d_{n}-1, s_{n}-1\right)$. In this situation it should be made clear that no vehicle will be selected to turn again until it completes the selected turn.

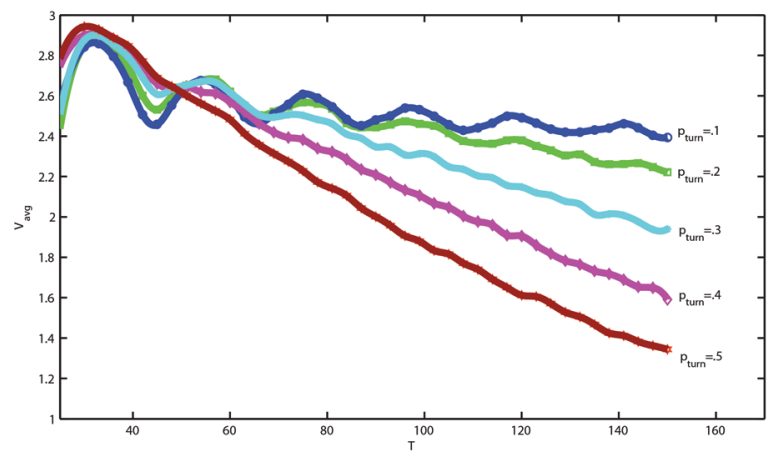

Figure 3. The average velocity is plotted versus the light cycle length for $p_{\text {slow }}=.1$, various turning probabilities, and $d_{x}=d_{y}=101$ for $\rho=.05$. Each point resembles the average velocity after aggregating the model for 125,000 steps.

Lastly, consider $s_{n}<v_{\text {turn }}$. In this situation the vehicle is close enough to the intersection to complete the turn in one time step provided $s_{n}<\min \left(v_{n}, v_{\text {turn }}, d_{n}-1, \widetilde{d}_{n}-1\right)$. The Turning Flag is turned on when a car is making a turn in a single time step, and must be moved onto the cross street in step 4 with special considerations. The position of an eastbound car turning northbound will be $a_{i j} \rightarrow a_{\mu v}$ where $\mu=i+s_{n}$ and $v=j-\left(v_{n}-s_{n}\right)$, where sn is the distance to the turning intersection. Similarly for a northbound car turning eastbound $\mu=i+\left(v_{n}-s_{n}\right)$ and $v=j+s_{n}$. In no situation is turning allowed when the light is currently red.

A vehicle will enter the intersection only if there is room for it. This modification over the BML model was introduced ${ }^{5}$ to attenuate the transition to a grid-locked state by restricting movement into the intersection, hence preventing blockage of the perpendicular lane of traffic.

In step 3 the vehicles randomly brake with randomization parameter $p_{\text {slow }}$. This models the effect of random slowing due to different behavioral patterns of drivers. Updating the position of each car is done in step 4. 
The Nagel-Schreckenberg model ${ }^{12}$ can be realized by setting $p_{\text {slow }}=0, N_{x}=1$, and $N_{y}=0$. Upon removal of $(a)$ in the second step and setting $p_{\text {turn }}=0$ the model reduces to Brockfeld et al.'s model. ${ }^{5}$ Adding turning to a basic CA traffic model allows us to better analyze the specific effect of turning on the flow of traffic. The influence of the new parameter $p_{\text {turn }}$ is addressed in the following section.

\begin{tabular}{|c|r|r|r|r|}
\hline$\epsilon / \rho$ & .05 & .10 & .15 & .20 \\
\hline .1 & $(444.537,-0.892)$ & $(401.091,-0.997)$ & $(403.052,-0.997)$ & $(513.239,-.943)$ \\
\hline .2 & $(217.572,-0.898)$ & $(231.090,-.881)$ & $(203.920,-.988)$ & $(250.989,-.997)$ \\
\hline .3 & $(141.552,-.857)$ & $(142.404,-.846)$ & $(134.436,-0.992)$ & $(141.059,-.877)$ \\
\hline .4 & $(106.427,-.860)$ & $(106.427,-.860)$ & $(101.720,-0.983)$ & $(104.620,-.945)$ \\
\hline .5 & $(85.853,-.898)$ & $(86.266,-.855)$ & $(81.759,-.952)$ & $(78.386,-.945)$ \\
\hline
\end{tabular}

Table 1. $(K, \eta)$ Values

It is reasonable to expect that introducing turning will reduce the average velocity $V_{\text {avg }}$ compared with the turn-free situation. In essence a vehicle selected for turning behaves similar to that of one approaching a red light, slowing down as the vehicle approaches it. Theoretically this is easily realized. Consider $p_{\text {turn }}=p_{\text {slow }}=0$, it is straight-forward to verify that

$$
\lim _{T \rightarrow \infty} V_{\text {avg }}=\min \left(\frac{1-\rho}{2 \rho}, \frac{v_{\max }}{2}\right):=V_{\text {steady }}
$$

This is in contrast to the case when $p_{\text {turn }}>0$ as for sufficiently long enough light cycles all the cars will eventually turn on the perpendicular road and come to a stop at the next red light. In other words for $p_{t u r n}>0$ the $\lim _{T \rightarrow \infty} V_{a v g}=0$. This is indicative of a critical light cycle length for which the average velocity may decrease sharply. In either case, it is suggestive that one may find a critical length cycle $T_{\epsilon}$, such that $V_{\text {avg }}<\epsilon V_{\text {steady }}$ for a prescribed $\epsilon>0$. In other words, one may determine the light cycle length that will only lower the average speed by $50 \%$ compared to the steady-state, turn-free, velocity. This motivates our exploration into a power law that depends on $\rho$ and $p_{\text {turn }}$ for a prescribed $\epsilon$. 


\section{RESULTS - A POWER LAW}

In the given calculations the simplest network is investigated with only one east and north bound intersection with periodic boundary conditions. The turning velocity is held constant at 1 . The maximum velocity $v_{\max }$ is set to 5 . The length of the road is 101 cells, i.e. $d_{x}=d_{x}=100$. For each value of the light cycle length, density, and probabilities of slowing and turning the model is aggregated for 125,000 time steps. Upon completion the average velocity is calculated by averaging the the calculated velocities at each time-step. A typical plot for $p_{\text {slow }}=.1$ and $\rho=.05$ is shown in Fig. 3 .

The value of $p_{\text {turn }}$ has a considerable influence on the average velocity for each simulation. For extremely small probabilities the average velocity has the same fundamental characteristics as in the $p_{\text {turn }}=0$ case (see Fig. 3). At large densities $\rho>.5$ jamming can occur provided the light cycle length is long enough and $p_{\text {turn }}>0$. For illustration let the light be green for eastbound(northbound) traffic and $p_{\text {slow }}=0$. Turning vehicles onto the northbound(eastbound) lane will be forced to slow down as a result of the upcoming traffic light. If the light cycle is long enough the vehicles will aggregate to the northbound(eastbound) lane. This will occur when the light cycle is longer than the number of time-steps it takes for all the eastbound( northbound) cars to be selected for turning $\left(\frac{1}{p_{u u m}}\right)$ and complete the turn at the next intersection, i.e.

$$
T>T^{*}=\frac{1}{p_{\text {turn }}}+\min \left(d_{x}, d_{y}\right) . \quad \text { Equation } 1 .
$$

For $T>T^{*}$ and $\rho>.5$ the northbound (eastbound) lane will come to a complete stop preventing eastbound (northbound) cars from moving through the intersection. The jam will persist till the light changes and the car blocking the intersection is selected again for turning. In reality for even small values of $p_{\text {turn }}>0$ and $\rho$ is low as . 2 jamming may occur for $T<<T^{*}$. It is reasonable to compare the observed jamming to real-life situations when a car sits in the intersection as a result of heavy traffic consequently interfering the perpendicular flow of traffic. This may suggest that for heavy traffic long light cycle lengths $\left(T>T^{*}\right)$ should be avoided as a deterrent to the formation of jams. The persistence of jamming appearing at densities as low as .2 suggests that a synchronous lighting strategy may not be ideal outside of low density traffic.

To avoid jamming caused by the introduction of turning, densities less than .2 will be considered only. In addition, to focus exclusively on the effect of turning, $p_{\text {slow }}$ will be set to zero in the following calculations. The algorithm is simulated at different densities and values of $p_{\text {turn }}$. $v_{\text {turn }}$ remains at one, and $v_{\max }=5$. The amplitude of the oscillations observed in the turnfree case are reduced and vanish as the turning probability increases (see Fig. 3). The light cycle length, $T_{\epsilon}$, for which the average velocity is first less than, $\epsilon V_{\text {steady }}$ is determined. It is clear that this satisfies a power law distribution that depends on $\rho$ and $p_{\text {turn }}$ (see Fig. 4 for the $\rho=.1$ case). More precisely,

$$
T_{\epsilon}\left(p_{\text {turn }}, \rho ; \epsilon\right)=K p_{\text {turn }}^{\eta}, \quad \text { Equation } 2 .
$$




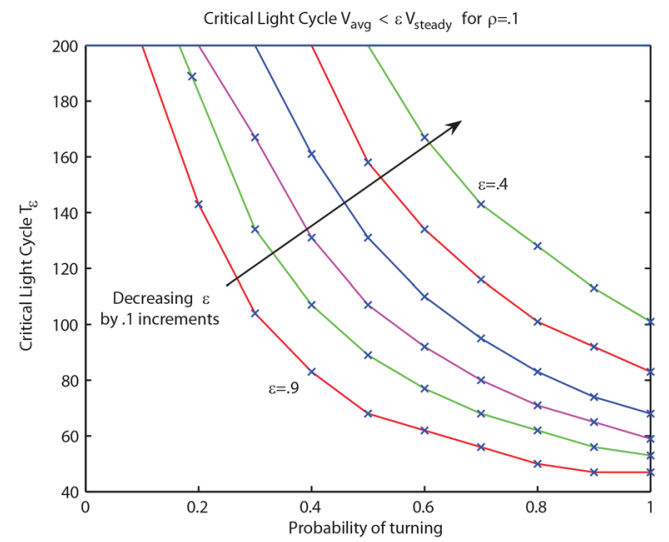

Figure 4. $T_{\epsilon}$ is calculated numerically for $\epsilon=.5, \rho=.1, d_{x}=d_{y}=101$, and $p_{\text {slow }}=0$.

The value of $p_{\text {turn }}=.1, .2, \ldots, 1$.

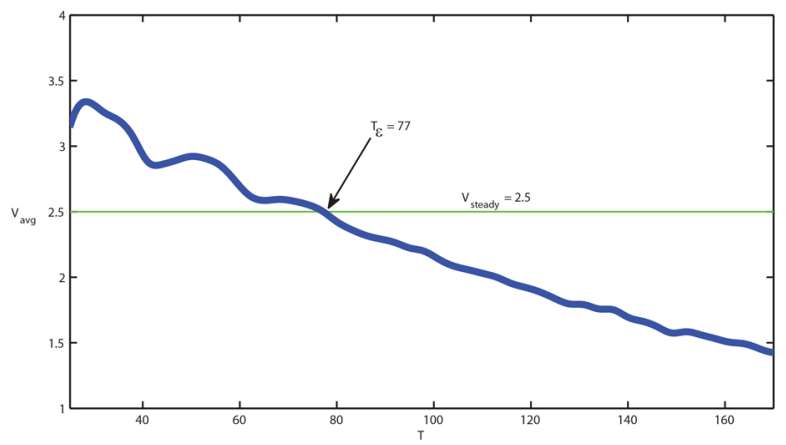

Figure 5. $T_{\epsilon}$ is shown for $\epsilon=1, \rho=.05, d_{x}=d_{y}=101, p_{\text {turn }}=.4$, and $p_{\text {slow }}=0$.

Notice for values $T>T_{\epsilon}$ the average velocity continues to decrease.

The experimental value of $T_{\epsilon}$ is 77 . Using Eq. 3 predicts a value of 75.67.

where $K$ and $\eta$ are functions that depend on $\rho$ and $\epsilon$. The $(K, \eta)$ coordinate pair for $\rho=.05, .1, .15$, and .2 are shown in Table I. The function $K$ also obeys a power law of the form $K_{0}(\rho) \epsilon^{\eta 0(\rho)}$. The function $\eta$ is fitted to a plane in a least squares sense. This results in a more general power law

$$
T_{\epsilon}\left(p_{\text {turn }}, \rho ; \epsilon\right)=\left(m_{k} \rho+b_{k}\right) \epsilon^{m \eta \rho+b \eta} p_{\text {turn }}^{a \epsilon+b \rho+c}
$$

Equation 3.

where $m_{k}, b_{k}, m_{\eta}, b_{\eta}, a, b$, and $c$ are constants are shown in Table II.

\begin{tabular}{|c|r|}
\hline$m_{k}$ & -24.76345201312850 \\
\hline$b_{k}$ & 42.89042951253280 \\
\hline$m_{\eta}$ & -0.50559741111383 \\
\hline$b_{\eta}$ & -0.98871563734671 \\
\hline$a$ & 0.06225834560947 \\
\hline$b$ & -0.22611688813737 \\
\hline$c$ & -0.88937638486533 \\
\hline
\end{tabular}

Table 2. Least Squares Coefficients for Eq. 3 
As an example, Fig. 5 shows the average velocity for various light cycle lengths. In the case of $\epsilon=1$ the average velocity is less than $V_{\text {steady }}$ once $T>77$. Using Eq. 2 and the coefficients found in Table I the predicted value is 75.67. Clearly, these are comparable results.

Fig. 6 shows $\epsilon$-slices as predicted from the theory for $\rho=.05$ compared with experimental results. For example, for a $40 \%$ turning probability Eq. 3 predicts that light cycle lengths greater than 200 will generate a $50 \%$ reduction in the average velocity.

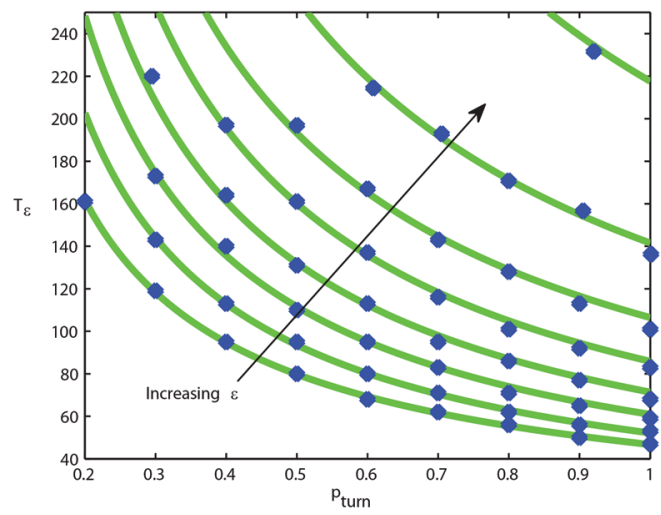

Figure 6. Experimental computation of $T_{\epsilon}$ versus Eq. 3 for $\rho=.05$ and $\epsilon=.9, \ldots, .1$. The maximum least squares error is 0.0096228 clearly showing good agreement.

\section{DISCUSSION}

A simple model for traffic flow was introduced borrowing ideas from previous models. ${ }^{5}$ It is shown that persistent jamming can occur as a result of turning, even for densities that are rather low. In fact, jamming is guaranteed for moderate densities with sufficient light cycle lengths. Therefore it is a synchronous lighting strategy which should be avoided in these situations.

In the analysis of lower densities it was shown that the average velocity is reduced by cars turning onto cross streets. In particular, it was shown that a power law depending on the density and the probability of turning is found that can predict when the average velocity is lower than $\epsilon V_{\text {steady }}$ for all $T>T_{\epsilon}$. It is clear that a traffic controller using a synchronous lighting strategy should chose a light cycle length sufficiently less than $T_{\epsilon}$ to avoid large reductions in the flow of traffic. 


\section{REFERENCES}

[1] Aw, A., and Rascle, M. (2000) Resurrection of "second order" models of traffic flow, SIAM Journal on Applied Mathematics 60, 916-938.

[2] Bellomo, N., Delitala, M., and Coscia, V. (2002) On the mathematical theory of vehicular traffic flow i. fluid dynamic and kinetic modelling, Mathematical Models and Methods in Applied Sciences 12, 1801-1843.

[3] Biham, O., Middleton, A. A., and Levine, D. (1992) Self-organization and a dynamical transition in traffic-flow models, Phys Rev A 46, R6124-R6127.

[4] Brenneis, A., Konhauser, P., and Kerner, B. (1995) Comparison of one and two equation models for traffic flow calculations, In In Proc. 26th AIAA Fluid Dynamics Conference, San Diego, CA.

[5] Brockfeld, E., Barlovic, R., Schadschneider, A., and Schreckenberg, M. (2001) Optimizing traffic lights in a cellular automaton model for city traffic, Phys Rev E Stat Nonlin Soft Matter Phys 64, 056132.

[6] Chowdhury, D., Santen, L., and Schadschneider, A. (2000) Simulation of vehicular traffic: a statistical physics perspective, Computing in Science and Engineering and Science 2, 80-87.

[7] Chowdhury, D., Santen, L., and Schadschneider, A. (2000) Statistical physics of vehicular traffic and some related systems, Physics Reports 329.

[8] NH, G., and Stamatiadis, C. (2009) Traffic networks, optimization and control of urban, In Encyclopedia of Complexity and Systems Science, pp 9470-9500.

[9] Helbing, D. (1995) Improved fluid-dynamic model for vehicular traffic, Phys Rev E Stat Phys Plasmas Fluids Relat Interdiscip Topics 51, 3164-3169.

[10] Kerner, B. (2004) The Physics of Traffic, Springer.

[11] Klar, A., Kuhne, R., and Wegener, R. (1996) Mathematical models for vehicular traffic, Surveys Math. Indust 6, 215-239.

[12] Nagel, K., and Schreckenberg, M. (1992) A cellular automaton model for freeway traffic, J Phys I 2, 2221-2229.

[13] Payne, H. (1971) Models of freeway traffic and control, Math. Models Publ. Sys 1, 51-61.

[14] Prigogine, I. (1961) Theory of traffic flow, A Boltzman like approach to the statistical theory of traffic flow, Elsevier Publishing Company.

\section{ABOUT THE STUDENT AUTHOR}

Tracy Finner worked on this project during her senior year (2010-2011) at the University of Arizona. She finished her M.Sc. in industrial engineering in the spring of 2014. She is now an Industrial Engineer at Raytheon.

\section{PRESS SUMMARY}

Traffic flow models are central to urban planners and developers. In this paper, a traffic flow model is proposed that models the interaction between individual vehicles and subsequent driver behavior to the traffic light system. The model is then used to develop empirical evidence for a statistical correlation between the density of vehicles, frequency of turning vehicles, and the timing of the traffic signal. 


\title{
On Deflection of Potentially Dangerous Asteroids
}

\author{
Josh Fixelle and Mikhail Kagan* \\ Department of Science and Engineering, Pennsylvania State University, Abington \\ *Mentor:mak411@psu.edu
}

\begin{abstract}
As has been widely discussed recently, our planet may become a target for asteroids. We consider several scenarios proposed to prevent asteroid collisions with Earth. The asteroid 99942 Apophis is considered as a typical representative. Among others, the recent "gravitational tractor" scenario ${ }^{2}$ is discussed. For a simplistic toy-model we obtain estimates for both the mass of the tractor and the amount of fuel required to tow a potentially dangerous asteroid off-course so as to avoid a collision with the Earth. In addition, we analyze two more scenarios titled "sling-shot" and "bumping", and comment on their relative efficiency compared to the "towing" scenario. Based on the analysis, the bumping scenario looks most promising.
\end{abstract}

\section{KEYWORDS}

Near-Earth objects, Gravitational Tractor, Asteroid 99942 Apophis

\section{INTRODUCTION}

After watching a presentation on the possibility of asteroids colliding with the planet Earth, a thought occurred: this may be reality in the near future. In fact, due to one specific asteroid (99942 Apophis), this idea may be even more of a reality than first thought. Situations such as an asteroid impact have been depicted in disaster films since the late twentieth century, all with the same solution - to destroy the asteroid with high yield explosives. There is however an issue with the use of explosives: an asteroid has an original quantity of energy (kinetic, potential, etc.). If the asteroid were to be split into smaller pieces (as an explosion would), then the only change occurring is that the overall energy is then concentrated throughout several pieces instead of just one, and thus after collision the energy is spread over several impact locations as opposed to concentrated at one. Another consideration is that of aerodynamic heating. Most small space born objects collide with the Earth's atmosphere at high velocities, which in turn generates friction between the object and the atmosphere - this friction will tend to disintegrate most of the materials that enter the Earth's atmosphere.

With that said, the small asteroid pieces - resulting from the explosive device - may or may not disintegrate (depending on their volume) upon entry. This aerodynamic heating may result in anything from minimal heating of the Earth's atmosphere, to a more notable heating - which could in turn create meteorological changes throughout the planet. One may also consider the fact that small asteroid pieces - the ones that do not disintegrate upon reentry - may still cause significant damage (consider if one were to impact in the center of a large city). When the asteroid is broken up, the initial energy remains the same (with the addition of the energy from the explosion), and thus will still be transferred to the Earth upon collision (over a larger area, and in multiple forms) - the exception to this would be considering a transfer of momentum to pieces due to the explosion, giving them a trajectory that does not collide with the Earth. These non-colliding pieces however, would be insignificant when compared to the amount that would in fact still collide with the Earth. With that said, we realized that as a planet we currently have no viable means to "defend" against a possible asteroid collision.

\section{AJUR Volume 12 | Issue 1 | August 2014}




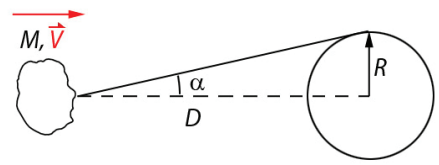

Figure 1. Asteroid of mass $M$ moving at speed $V$ is approaching the Earth. A safe scenario must ensure that the asteroid will be deflected sideways by the minimum angle $\alpha=\frac{R}{D}$, where $R$ is the radius of the Earth, and $D$ is the intercept distance.

A typical simplistic problem can be formulated as follows. Consider a potentially dangerous asteroid on a collision course with the Earth, as illustrated in Fig.(1). The asteroid has mass $M$, velocity $\vec{V}$ and, in the worst case scenario, zero impact parameter, that is its extended trajectory passes through the center of the Earth. The latter implies that the asteroid should be deflected sideways by at least one radius of the Earth, $R$. Assuming that the asteroid is detected (and gets acted upon) at distance $D$ from the Earth, its trajectory must be deflected by a small angle $\alpha=\frac{R}{D}$. For the reader's convenience we summarize all the relevant numerical data in the table below, taking the 99942 Apophis as a typical representative. Footnote 1

\begin{tabular}{|l|l|}
\hline Physical quantity & Numerical Value \\
\hline Radius of the Earth, $R$ & $6.37 \cdot 10^{6} \mathrm{~m}$ \\
\hline Intercept distance, $D$ & $2.55 \cdot 10^{11} \mathrm{~m}$ \\
\hline Minimum deflection angle, $\alpha=\frac{R}{D}$ & $2.50 \cdot 10^{-5}$ \\
\hline Speed of the asteroid, $V$ & $3.07 \cdot 10^{4} \frac{\mathrm{m}}{\mathrm{s}}$ \\
\hline Travel time, $t=\frac{D}{V}$ & $8.31 \cdot 10^{6} \mathrm{~s} \approx 0.263$ years \\
\hline Mass of the asteroid, $M$ & $2.10 \cdot 10^{10} \mathrm{~kg}$ \\
\hline Gravitational constant, $G$ & $6.67 \cdot 10^{-11} \frac{\mathrm{m}^{3}}{\mathrm{~kg} \cdot \mathrm{s}^{2}}$ \\
\hline
\end{tabular}

Table 1. Typical numerical data relevant for a collision problem. The mass and speed of a typical asteroid correspond to those of 99942 Apophis.

Provided that the potentially dangerous asteroid is detected early enough and is intercepted sufficiently far away from the Earth, it needs to be deflected by just a tiny angle $\alpha \sim 10^{-5}$ (see Table 1) to prevent collision. This fact gave rise to a promising idea that there should a viable method of deflecting the asteroid on a collision course with the Earth (rather than using high yield explosives), by means that are available with current day technology. ${ }^{1}$

Footnote 1 In reality, 99942 Apophis follows a nearly circular orbit around the Sun and appears in a close proximity of the Earth once every seven years. In our toy model, however, we assume the potentially dangerous asteroid to be on a straight collision course with the Earth, and take the mass and speed of 99942 Apophis just for sake of estimate. In addition, we consider the problem in the reference frame associated with the Earth.

\section{AJUR volume 12 | Issue 1 | August 2014}




\section{SCENARIO 1. DEFLECTION BY GRAVITATIONAL PULL}

It was recently suggested ${ }^{2}$ that by flying a small spacecraft ('gravitational tractor') alongside an asteroid, the gravitational pull created from the spacecraft could in fact alter the trajectory of the asteroid, avoiding impact entirely.

In Fig. 2, a "gravitational towing" scenario is illustrated. A spacecraft is hovering near the asteroid at a constant distance $d$ (as measured from the center of the asteroid), thus creating a constant force of gravity acting on the asteroid in a sideways direction. The spacecraft's thrusters provide a force exactly needed to prevent the spacecraft from falling onto the asteroid. For simplicity, we neglect the pressure of the fuel exhaust on the surface of the asteroid. Below we investigate this scenario and provide some numerical estimates.

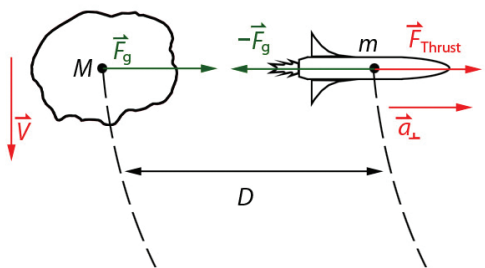

Figure 2. Scenario 1. Asteroid is being deflected by the gravitational pull exerted by a spacecraft of mass $m$ flying alongside at a constant distance $d$ away from the asteroid.

\subsection{ESTIMATION OF THE SPACECRAFT MASS}

The gravitational force on the asteroid provides acceleration

$$
a=\frac{G m}{d^{2}},
$$

Equation 1.

which results in a sideways displacement

$$
\Delta x=\frac{1}{2} a t^{2} .
$$

Here $t=\frac{D}{V}$ is the time it takes for the asteroid to reach the Earth (see Table 1). Setting the deflection equal to the radius of the Earth gives us an estimate for the minimum mass of the spacecraft

$$
m=\frac{2 R d^{2} V^{2}}{G D^{2}} \text {. }
$$

Equation 2.

Substituting the values from Table 1 and assuming that $d \approx 175 \mathrm{~m}$, we obtain

$m \approx 8.478 \cdot 10^{7} \mathrm{~kg}$.

For comparison, the mass of the 99942 Apophis asteroid is $2.1 \cdot 10^{10} \mathrm{~kg}$, whereas the mass of the NASA space shuttle is $2.03 \cdot 10^{6} \mathrm{~kg}$. With that said, the mass required to deflect an asteroid with the velocity of the 99942 Apophis asteroid with an impact parameter of zero is more than that of the NASA space shuttle. Thus the idea so far is implausible, as the mass required is around that of 37 NASA space shuttles (not including the fuel required). For the sake of completion, we will still continue the analysis. 


\subsection{ESTIMATION OF THE REQUIRED AMOUNT OF FUEL}

The next step in investigating the gravitational towing scenario is to determine if the amount of fuel (mass) required to accomplish this mission is remotely plausible. The most prevalent fuel requirement is that of the necessity to keep the spacecraft from colliding with the asteroid due to gravitational attraction. There is also the fuel requirement to place the spacecraft in orbit, and to send the spacecraft the distance required to intercept the asteroid, however for now those requirements can be assumed negligible. Since the separation between the asteroid and the spacecraft remains constant, in the reference frame associated with the Earth, the spacecraft is moving with the same acceleration as the asteroid. Therefore the thrust generated by the engines should exceed the gravitational force

$$
F=\frac{G M m}{d^{2}}
$$

by $m a$, where $a$ is the acceleration from Eq.(1). In other words, the engines should provide the force

$$
F^{\prime}=\frac{G(m+M) m}{d^{2}} \approx \frac{G M m}{d^{2}}
$$

The latter is given by

$$
F^{\prime}=v_{\text {rel }}\left|\frac{d m}{d t}\right|,
$$

where $v_{\text {rel }}$ is the exhaust speed of the fuel, and $\left|\frac{d m}{d t}\right|$ is the mass rate. Using again the time from Table 1, we obtain an estimate for the total amount of fuel needed to tow the asteroid off-course

$$
\begin{aligned}
\Delta m & =\left|\frac{d m}{d t}\right| \frac{D}{V} \\
& =\frac{G M m}{d^{2} v_{r e l}} \frac{D}{V} .
\end{aligned}
$$

Equation 3.

Assuming the minimum mass found in Eq.(2), the mass of the required fuel can be written as

$$
\Delta m=2 \alpha M \frac{V}{v_{\text {rel }}} \text {. }
$$

Equation 4.

To estimate the amount of fuel we take the exhaust speed for the J-2s engine,

$v_{\text {rel }}=4.28 \cdot 10^{3} \mathrm{~m} / \mathrm{s}$, which yields

$\Delta m \approx 7.53 \cdot 10^{6} \mathrm{~kg}$.

The latter is approximately four times that of the mass of the NASA space shuttle, and still less than the mass of the spacecraft itself. In other words, this idea is plausible if the mass of the spacecraft was reasonable, however due to the enormous mass of the spacecraft and the large mass of the fuel required to maintain the distance of separation between the spacecraft and asteroid, the idea is not plausible.

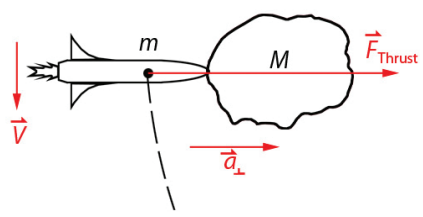

Figure 3. "Pushing scenario". Asteroid is being deflected off-course by the engines a spacecraft landed on its surface. 


\subsection{COMPARISON WITH THE "PUSHING SCENARIO"}

The towing scenario discussed above was suggested, ${ }^{2}$ as a less technically demanding way of deflecting asteroid off its collision course. The advantage being is that the tractor is insensitive to the structure of the asteroid but its mass. Moreover, it avoids landing on the surface of the asteroid which is one of the most challenging cosmic tasks. In addition, even if the landing has been successful, rotation of the asteroid makes it extremely difficult to direct the thrust, as well as merely to control the (unmanned) spacecraft, as it might get out of the line of sight.

Suppose, however, that the challenges associated with landing the spacecraft on the asteroid and navigating it have been successfully overcome (see Figure 4 ). What would be the required specifications of the spacecraft and the amount fuel to deflect the asteroid off-course by the same amount as before? We start by noting that now the spacecraft and the asteroid constitute one system, thus subjects to one common force provided by the spacecraft's engines. Clearly, only the combined mass, $M+m$, now matters, in which the mass of the spacecraft can be neglected, as $m \ll M$. Moreover, since the relative speed of the exhausted fuel $v_{r e l}$ is much greater than the sideways velocity of the system, the total sideways impulse provided by the engines can estimated as

$$
\Delta P_{\perp} \approx \Delta m v_{\text {rel }} \text {. }
$$

On the other hand, a successful deflection would require a sideways momentum of the asteroid of approximately

$$
\Delta P_{\perp}=\alpha P_{\|} \equiv \alpha M V .
$$

The latter leads to the following estimate for the mass of the fuel

$$
\Delta m=\alpha M \frac{V}{v_{r l}} . \quad \text { Equation } 5 .
$$

This expression gives a generic estimate for the amount of the required fuel regardless the details of a particular "towing" or "pushing" scenario. It is easy to see that the above result has the same order of magnitude as in Eq. (4).

To clarify the seeming discrepancy in the numerical prefactor between Eqs.(4) and (5), we should note that providing the asteroid with a transversal momentum $P_{\perp}=\alpha P_{\|}$is not yet sufficient to deflect it by an Earth radius $R$, unless the impulse is impeded over a very short period of time (much shorter than the travel time) in the beginning of the deflection phase. A scenario that realizes this idea is discussed in the next section.

For a long-term "pushing" or "towing", one needs to approach the problem along the lines of the previous subsection by considering the (constant) sideways acceleration as in Eq.(1). It is then easy to see that the corresponding thrust force should be exactly the same as that required in the previous subsection to prevent the spacecraft from falling onto the asteroid. Indeed, when computing the total external (towing) force acting upon the spacecraft-asteroid system (see Fig. 2), the two gravitational forces mutually cancel yielding the same resultant force.

We thus arrive at the conclusion that the only advantage of the "pushing" scenario is that it may work with a lighter spacecraft, as its mass would be irrelevant. It is, however, impossible to safe fuel. 


\section{SCENARIO 2. SLING-SHOT DEFLECTION.}

The main idea behind this scenario is to "sling-shot" a spacecraft over the asteroid. If the spacecraft passes close enough to the asteroid (i.e. the spacecraft's impact parameter $\rho$ is sufficiently small) due to gravitational attraction the asteroid will also be deflected off its collision course. Provided that the asteroid needs to be deflected a very small angle $\alpha \sim 10^{-5}$, the scenario may turn out to be quite realistic.

The "sling-shot" scenario is illustrated in Fig.4. On the left is the scattering as viewed in the reference frame associated with the asteroid (A-frame). The spacecraft approaches from the bottom with initial speed $v_{\infty}$ and impact parameter $\rho$. It then undergoes elastic Coulomb scattering by angle $\theta$, so that the final speed of the spacecraft far away from the asteroid is also equal $v_{\infty}$. The same process as viewed in the laboratory frame (L-frame), e.g. heliocentric frame, is depicted on the right of Fig.4. The asteroid scatters by a small angle $\alpha$ and receives a transversal velocity $\vec{V}_{\perp}$ and momentum

$\Delta P_{\perp}=\alpha P_{\|}=\alpha M V$

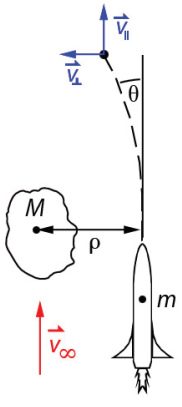

Equation 6.

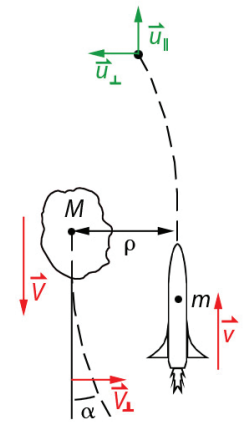

Figure 4. Scenario 2 . The spacecraft is "sling-shot" over the asteroid so as to deflect it by gravitational attraction. The left image is in the frame of the asteroid (A-frame), and the right image is in a laboratory frame, e.g. heliocentric frame (L-frame).

which is equal to the transversal momentum of the spacecraft. Rigorously speaking, the A-frame is not inertial. However, given that typical changes in the asteroid's velocity, hence its acceleration, are small (of relative order of $\alpha$ ) compared to the typical speeds, the A-frame can be considered inertial with the required accuracy. Furthermore, up to a negligible amount, the transversal momentum of the spacecraft after collision is the same in both frames and equals that in Eq.(6).

In the light of this, a successful sling-shot scenario would require maximization of the transversal impulse received by the spacecraft during the scattering. Given fixed $v_{\infty}$ and $\rho$, the maximum transversal impulse $\Delta p_{\perp}$ can be attained if the spacecraft is launched at initial angle $\theta / 2$ to the right from the vertical. In that case, it will scatter at angle $\theta / 2$ to the left from the vertical, resulting in

$$
\Delta p_{\perp}=m \Delta v_{\perp}=m v_{\infty}\left(2 \sin \left(\frac{\theta}{2}\right)\right)
$$

It is easy to see that the situation depicted in Fig. 4 would correspond to a factor of $\sin \theta$ instead of the one in the parenthesis and lead to a smaller transversal impulse. Footnote 2

Footnote 2 Clearly, $\frac{\sin \theta}{2 \sin (\theta / 2)}=\cos (\theta / 2)<1$. 
The scattering angle is given by ${ }^{3}$

$$
\tan \left(\frac{\theta}{2}\right)=\frac{G M}{\rho v_{\infty}^{2}} \quad \text { Equation } 8 .
$$

Using Eq.(7) and the inequality $\sin (\theta / 2)<\tan (\theta / 2)$, we obtain the following upper bound on the transversal impulse

$$
\Delta p_{\perp}<2 \frac{G m M}{\rho v_{\infty}}=m \frac{v_{c s c}^{2}}{v_{\infty}},
$$

where $v_{e s c}=\sqrt{\frac{2 G M}{\rho}}$ is the escape velocity from the asteroid. Obviously, $v_{\infty}$ must be greater than vesc, which implies that

$$
\Delta p_{\perp}<m v_{e s c}
$$

For the asteroid at hand, the mass and the impact parameter are of order $M \sim 10^{10} \mathrm{~kg}$ and $\rho \sim 100 \mathrm{~m}$ respectively (see Table 1), which corresponds to an escape velocity of order of $0.1 \mathrm{~m} / \mathrm{s}$. Clearly, the bound above makes it impossible for the spacecraft to have a sufficient change in transversal momentum of order of $\alpha M V \sim m V$, with the required speed $V \sim 10^{4} \mathrm{~m} / \mathrm{s}$. Let us remark on this result from a different perspective. As estimated above, the minimum transversal impulse of the asteroid should be such that the spacecraft's sideways speed is of order of the asteroid's speed $V \sim 10^{4} \mathrm{~m} / \mathrm{s}$. While this is a realistic value for the spacecraft's speed, the transfer of the corresponding sideways momentum to the asteroid requires strong enough interaction between the bodies. In other words, the deflection would be successful if the spacecraft simply (inelastically) hit the asteroid sideways and transferred the needed impulse through contact forces. As the analysis above shows, the transfer cannot be realized at a sufficient rate if the interaction is purely gravitational. We investigate the "bumping" possibility in the next section.

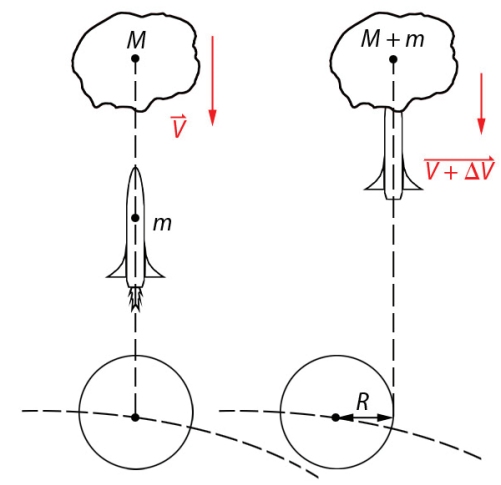

Figure 5. Scenario 3. Asteroid is moving in the ecliptic plane towards the Sun so that it would reach the Earth's orbit exactly when the Earth is on the line Sun-Asteroid. 


\section{SCENARIO 3. "BUMPING"}

Another promising collision avoidance possibility is associated with slowing the asteroid down rather than deflecting it off-course. From now on we consider the heliocentric reference frame and suppose, for simplicity, that the asteroid is approaching the orbit of the Earth along a radial direction (see Fig. 5). Initially, the asteroid is at distance $D$ away from the Earth's orbit and is moving with velocity $V$ towards the Sun. If no action is undertaken, the asteroid will collide with the Earth at the intersection of the orbit with the line Sun-Asteroid.

Unlike the previous sections, timing is critical in this scenario. If the asteroid crosses the Earth's orbit later (or earlier), the collision would be avoided. In fact, it would be sufficient if the asteroid is delayed by an amount of time, $t_{\perp}$, such that the Earth would have traveled at least one its radius, $R$. Specifically the minimum delay is given by

$$
t_{\perp}=\frac{R}{V_{\perp}}
$$

where $V_{\perp}$ is the Earth's orbital speed. It is easy to see that the delay is much less than the travel time. Indeed,

$$
\frac{t_{\perp}}{t}=\frac{R}{D} \frac{V}{V_{\perp}} \approx \alpha \ll 1 .
$$

In the last approximation we have used the fact that $V_{\perp} \approx V$.

Relative smallness of the delay time allows to simplify the further analysis. In particular, delaying the asteroid by $t_{\perp}$ requires slowing it down by $\Delta V$ such that

$$
\frac{\Delta V}{V}=\frac{t_{\perp}}{t}=\alpha \frac{V}{V_{\perp}} .
$$

The slowing down can be realized by using the "Bumping" scenario, that is by placing a relatively small obstacle of mass $m$ on the asteroid's way. After the collision with the bump, the fractional decrease in the asteroid's speed would be equal to $m / M$. Therefore, the mass of the obstacle, should constitute the following fraction of the asteroid's mass:

$$
\frac{m}{M}=\frac{\Delta V}{V}=\frac{t_{\perp}}{t}=\alpha \frac{V}{V_{\perp}} . \quad \text { Equation } 9 .
$$

For Apophis, the minimum required mass of the bump would be

$$
m \approx \alpha M=5.25 \cdot 10^{5} \mathrm{~kg} \text {, }
$$

which is much smaller than that in the previous scenarios. 


\section{DISCUSSION}

We have considered several possible scenarios for deflecting a potentially dangerous asteroids off their collision course with the Earth and estimated the minimum required mass of the spacecraft, as well as the amount of fuel needed during the deflecting stage. Remarkably, the mass of the fuel (when applicable) and the mass of the "bump" in the bumping scenario turned out to be of the same order of magnitude given by

$$
m \approx \alpha M \frac{V}{V_{\perp}},
$$

where $\alpha$ is the deflection angle, $M$ and $V$ are the mass and speed of the asteroid. The denominator, $V_{\perp}$, is either the exhaust speed of the fuel (in the scenarios involving engines) or the sideways (orbital) speed of the Earth. Typically, the mass of the spacecraft (in the corresponding scenarios) is by an order of magnitude greater than that of the fuel. In the light of this, the bumping scenario looks much simpler and cheaper, as it only requires placing the bump at the "appropriate" location. Although that might be a non-trivial task, it still avoids many complications associated with spacecraft maneuvering. Although the gravitational towing scenario may be efficient in deflecting an asteroid away from a "key-hole", it is less so when a deflection by a radius of the Earth is required.

In order to minimize the mass of the fuel in the engines-based scenarios, one should aim at a greater $V_{\perp}$. In that sense, engines with greater $v_{r e l}$, but not necessarily with greater mass rate $\frac{d m}{d t}$, would be preferred. For instance the VASIMR engine currently being developed by Ad Astra Rockets has a propellant velocity measured as high as $2 \cdot 10^{4} \mathrm{~m} / \mathrm{s}$ and a mass rate of $0.07 \mathrm{~g} / \mathrm{s} .{ }^{4}$ Granted, with these current specifications, multiple engines would be needed, however, they are close to achieving what would be needed.

Comparative analysis of the "towing" and "pushing" scenarios (see Lu and Love ${ }^{2}$ and Section 1.3) indicates that, in general, towing is more promising. Pushing, however may be more efficient in case of a weak gravitational pull. For there is no limit on the pushing force, while the towing force (force of gravity between the tractor and the asteroid) should not be exceeded by $F_{\text {thrust }}$, so as to not $v=$ break the "tow-line". We note, however, that in the two examples discussed above, $F_{G} \approx F_{\text {thrust }} \approx$ $100 \div 1000$ (newtons). A few additional remarks are in order. Lu and Love ${ }^{2}$ took into consideration the fact that the thrust must not interact with the asteroid's surface (i.e. the thrust must be vectored so that it does not exert a force on the asteroid's surface, thus ensuring that the distance of separation does not increase, or the force of gravity between the two bodies is not canceled out by the thrust). We however did not take this into consideration, which would mean that a slightly greater thrust would be needed, thus more fuel must be used. In addition, we did not consider the escape velocity of the asteroid. This further limits the type of thruster which can be used, as if the impulse of the thrusters is too great, the spacecraft will "escape" from the gravitational field of the asteroid, and thus nullify the Gravitational Tractor.

There is another issue with the scenario proposed by Lu and Love. ${ }^{2}$ The scenario requires an extremely long period of time for the tractor to actually be practical (i.e. the asteroid must be intercepted at a long distance, a distance which will most likely not be seen in respect to our solar system, and with current day technology, would not be feasible to reach). The scenario will mostly only work for a specific example such as that of the 99942 Apophis asteroid, and thus has limited application.

Another possible issue with this is the distance of separation. If we take into consideration the 99942 Apophis asteroid - with an estimated length of 320 meters - a distance of separation of 175 meters may not be sufficient. Consider the fact that most asteroids have some spin to them, and that the distance of separation is measured from the center of mass of both bodies. This means that depending upon the spin of the asteroid, the spacecraft at a distance of separation of 175 meters may be within the "sweep area" (the total volume of space - in reference to the asteroids center of mass - occupied by the asteroid due to spin as $t \rightarrow \infty$ ), and thus may result in a collision. To correct 
for this, a larger mass of the spacecraft would be needed, and consequently more fuel, stretching the plausibility of the scenario even further.

Some other deflection ideas to test include the following: One of JPL's suggestions is to simply land a rocket on the surface of the asteroid, and fire its rockets, so that it changed the perpendicular momentum enough to divert it from impacting earth. This along with other "momentum" ideas could be theoretically tested. Besides direct contact, if for example a few massive crafts were fired at the side of the asteroid, that could change the perpendicular momentum. Or, if a high yield explosive is detonated next to the asteroid (not in it), it may transfer enough momentum to the asteroid to deflect it sufficiently. Perhaps consider the idea of the gravitational tractor, and the slingshot effect in terms of the electromagnetic force instead of the gravitational force. If the asteroid was magnetized, and a spacecraft with a super electromagnet was flown with a parallel trajectory, it could attract the asteroid, and they could "meet" (collide), at some point where the impact parameter of the asteroid is now $R$. Also, if the asteroid could be magnetized, the same example as just stated could be used, except instead of destroying the spacecraft, just use it to alter the asteroid's perpendicular momentum more, and perhaps be more efficient. Those two ideas are inspired by the fact that, on general grounds, it is easier to arrange for a stronger electromagnetic interaction than the gravitational one.

\section{ACKNOWLEDGEMENTS}

We thank Dr. Ann Schmiedekamp for helpful discussions and extremely useful information about potentially dangerous asteroids, Dr. Carl Schmiedekamp for pointing out the comprehensive review in Yeomans, ${ }^{1}$ and Dr. Moylan for the discussions on Coulomb scattering. The work of J.F. was generously supported by the ACURA fund of Penn State Abington.

\section{REFERENCES}

[1] Yeomans, D. (2009) Deflecting a Hazardous Near-Earth Object, In Proceedings 1st IAA Planetary Defense Conference: Protecting Earth from Asteroids, Granada, Spain.

[2] Lu, E. T., and Love, S. G. (2005) Gravitational tractor for towing asteroids, Nature 438, 177-178.

[3] Landau, L., and Lifshitz, E. (1976) Theoretical Mechanics, 3rd ed., Butterworth-Heinemann.

[4] Squirel, J. (2008) VASIMR Performance Measurements at Powers Exceeding $50 \mathrm{~kW}$ and Lunar Robotic Mission Applications, In International Interdisciplinary Symposium on Gaseous and Liquid Plasmas, Akiu/Sendai, Japan.

\section{ABOUT THE STUDENT AUTHOR}

Joshua Fixelle graduated from the Pennsylvania State University in December, 2013 with BS degrees in Astronomy \& Astrophysics and Engineering Science \& Mechanics (minors in Mathematics and Physics). He is currently living in Evanston, IL, while attending Northwestern University pursuing his Doctorate in Astrophysics (PhD).

\section{PRESS SUMMARY}

As has been widely discussed recently, our planet may become a target for asteroids (the asteroid 99942 Apophis is considered as a typical representative). We considered several scenarios proposed to prevent asteroid collisions with Earth, including the "gravitational tractor", "sling-shot", and "bumping" scenarios. We analyzed and compared the scenarios in regards to their relative efficiency compared to the "gravitational tractor", and concluded that the bumping scenario looks most promising.

\section{AJUR volume 12 | Issue 1 | August 2014}



